\title{
Neuronal RAR $\beta$ Signaling Modulates PTEN Activity Directly in Neurons and via Exosome Transfer in Astrocytes to Prevent Glial Scar Formation and Induce Spinal Cord Regeneration
}

\author{
Maria B. Goncalves, ${ }^{1}$ Tony Malmqvist, ${ }^{1}$ Earl Clarke, ${ }^{1}$ Chantal J. Hubens, ${ }^{1}$ John Grist, ${ }^{1}$ Carl Hobbs, ${ }^{1}$ Diogo Trigo, ${ }^{1}$ \\ (D) Mårten Risling, ${ }^{2}$ Maria Angeria, ${ }^{2}$ Peter Damberg, ${ }^{3}$ Thomas P. Carlstedt, ${ }^{1}$ and Jonathan P.T Corcoran ${ }^{1}$ \\ ${ }^{1}$ The Wolfson Centre for Age-Related Diseases, King's College London, Guy's Campus, London SE1 1UL, United Kingdom, ${ }^{2}$ Experimental Traumatology, \\ Department of Neuroscience, Karolinska Institutet, Stockholm SE-17177, Sweden, and ${ }^{3}$ Karolinska Experimental Research and Imaging Centre, \\ Department of Clinical Science, Intervention and Technology, Karolinska Universitetssjukhuset, Stockholm SE-17177, Sweden
}

Failure of axonal regeneration in the central nervous system (CNS) is mainly attributed to a lack of intrinsic neuronal growth programs and an inhibitory environment from a glial scar. Phosphatase and tensin homolog (PTEN) is a major negative regulator of neuronal regeneration and, as such, inhibiting its activity has been considered a therapeutic target for spinal cord (SC) injuries (SCIs). Using a novel model of rat cervical avulsion, we show that treatment with a retinoic acid receptor $\beta(\operatorname{RAR} \beta)$ agonist results in locomotor and sensory recovery. Axonal regeneration from the severed roots into the SC could be seen by biotinylated dextran amine labeling. Light micrographs of the dorsal root entry zone show the peripheral nervous system (PNS)-CNS transition of regrown axons. RAR $\beta$ agonist treatment also resulted in the absence of scar formation. Mechanism studies revealed that, in RAR $\beta$-agonist-treated neurons, PTEN activity is decreased by cytoplasmic phosphorylation and increased secretion in exosomes. These are taken up by astrocytes, resulting in hampered proliferation and causing them to arrange in a normal-appearing scaffold around the regenerating axons. Attribution of the glial modulation to neuronal PTEN in exosomes was demonstrated by the use of an exosome inhibitor in vivo and PTEN siRNA in vitro assays. The dual effect of RAR $\beta$ signaling, both neuronal and neuronal-glial, results in axonal regeneration into the SC after dorsal root neurotmesis. Targeting this pathway may open new avenues for the treatment of SCIs.

Key words: exosome; PTEN; retinoid; spinal cord regeneration

Significance Statement

Spinal cord injuries (SCIs) often result in permanent damage in the adult due to the very limited capacity of axonal regeneration. Intrinsic neuronal programs and the formation of a glial scar are the main obstacles. Here, we identify a single target, neuronal retinoic acid receptor $\beta(\operatorname{RAR} \beta)$, which modulates these two aspects of the postinjury physiological response. Activation of RAR $\beta$ in the neuron inactivates phosphatase and tensin homolog and induces its transfer into the astrocytes in small vesicles, where it prevents scar formation. This may open new therapeutic avenues for SCIs.

\section{Introduction}

Spinal cord injury (SCI) often results in permanent paralysis, largely as a result of the failure of the injured axons to regenerate in the adult mammalian CNS. The major impediments to axonal regeneration are unfavorable neuron-intrinsic mechanisms and the glia scar, which not only represents a physical barrier (Wanner et al., 2013) in which axonal tips get trapped, but also creates

Correspondence should be addressed to Jonathan Corcoran, The Wolfson Centre for Age-Related Diseases, King's College, Hodgkin Blvd., Guys Campus, London SE1 1UL, UK. E-mail: jonathan.corcoran@kcl.ac.uk.

DOI:10.1523/JNEUROSCI.1339-15.2015

Copyright $\odot 2015$ Goncalves et al.

This is an Open Access article distributed under the terms of the Creative Commons Attribution License Creative Commons Attribution 4.0 International, which permits unrestricted use, distribution and reproduction in any medium provided that the original work is properly attributed. 
an inhibitory extrinsic milieu due to molecules that are secreted by its components (Shearer and Fawcett, 2001; Silver and Miller, 2004). Axonal regeneration is shaped by neuronal and glial cues and an interchange between them (Toy and Namgung, 2013; Williams et al., 2015), so an ideal drug would tackle this cross talk for therapeutic benefit.

We have studied here axonal regeneration in a rat SCI resulting from cutting and subsequently implanting the cut end of dorsal roots into the SC. This type of injury is clinically relevant and analogous to the intraspinal plexus lesions that often occur as a result of motorcycle accidents and cause major functional deficits. Centrally projecting sensory axons of the severed dorsal root regenerate in the peripheral division of the dorsal root, but are normally arrested at the transitional region between the PNS and the CNS, the dorsal root entry zone (DREZ), and fail to re-enter the spinal dorsal horn (Carlstedt, 1985). The functional consequence is obviously permanent loss of sensation. Successful therapy would unequivocally be attributed to regenerating dorsal root axons growing pass the injury zone and into the SC, thus restoring sensory function.

A major contributor to regenerative failure is a diminished intrinsic capacity of adult axons to grow, based largely on inactivity in the phosphoinositide 3-kinase (PI3K)/Akt/mammalian target of rapamycin (mTOR) pathway, which is negatively regulated by phosphatase and tensin homolog (PTEN) (Park et al., 2010).

Previous work has shown that retinoic acid receptor $\beta 2$ (RAR $\beta 2$ ) signaling stimulates axonal outgrowth (Wong et al., 2006; Agudo et al., 2010) and that this occurs via PI3K signaling. Retinoic acid (RA) plays an essential role in the survival and differentiation of the vertebrate CNS and stimulates both neurite number and length in embryonic neuronal types (Maden, 2001). In the adult, RA-responsive elements are locally activated in the regenerating rat nerve after peripheral nerve injury (Zhelyaznik et al., 2003). In embryonic DRG neurons, RAR $\beta 2$ mediates neurite outgrowth induced by RA (Corcoran et al., 2000) and, in adult spinal cord explants, where RAR $\beta 2$ is absent, exogenous RAR $\beta 2$ stimulates neurite outgrowth (Corcoran et al., 2002). Furthermore, the delivery of RAR $\beta 2$ to adult neurons induces axonal regeneration programs within injured neurons and encourages axonal growth in the inhibitory CNS (Yip et al., 2006). Here, we show that activation of RAR $\beta$ signaling after dorsal root injury inactivates neuronal PTEN by shifting membrane PTEN into the cytoplasm and also inducing increased release of PTEN in exosomes. Given the importance of exosomes in neuron-glia communication (Chivet et al., 2013) and that cell-to-cell biological activity has been reported for PTEN exported in exosomes (Putz et al., 2012), we have explored their role in axonal regeneration. We found that neuronal PTEN can modulate astrocyte activity via exosome transfer by preventing their exacerbated proliferation. This results in the absence of an astrocytic scar after SCI and favors axonal regeneration in that the astrocytes rearrange in sleeves or tunnels that encourage the regrowing axons to follow centrally.

In summary, we found that $\mathrm{RAR} \beta$ signaling alters intrinsic and extrinsic signaling pathways to induce true axonal regeneration of primary sensory neurons in the CNS after dorsal root rhizotomy and reimplantation into the SC, resulting in sensory and locomotor recovery. The RAR $\beta$ pathway modulates PTEN signaling directly in neurons and via neuron-glia exosome transfer to create a permissive environment for axonal regrowth.

\section{Materials and Methods}

Surgery and drug treatments. All rat experiments were approved by the local veterinarian and ethical committees and performed according to
UK Home Office regulations. In male Wistar rats (8 per treatment group for each set of experiments) C5-C8 and T1 dorsal roots were cut flush with the SC surface. The cut ends of the dorsal roots were subsequently introduced through slits in the pia mater and positioned superficially in the SC adjacent to where they had been cut. Rats were treated with vehicle or a specific RAR $\beta$ agonist, CD2019 (1 mg/kg, synthesized by Sygnature Chemical Services), or with the exosome inhibitor GW4869 (1 mg/kg; Cayman Chemical) (Dinkins et al., 2014) alone or in conjunction with the RAR $\beta$ agonist by intraperitoneal injection (i.p.) 3 times a week for 4 weeks. At week 2, primary sensory afferents were labeled by injection of the anterograde tracer biotinylated dextran amine (BDA) in the median nerve. Animals were culled after 2, 4, or 5 weeks of treatment. Rats were perfused transcardially with heparinized $0.9 \% \mathrm{NaCl}$ solution and $4 \%$ paraformaldehyde in $0.1 \mathrm{M}$ phosphate buffer. The cervical cords with attached DRGs were dissected, rapidly removed, and postfixed with $4 \%$ paraformaldehyde (in $0.1 \mathrm{~m}$ phosphate buffer) for at least $2 \mathrm{~d}$ at room temperature. Tissue was then embedded in paraffin wax and $5 \mu \mathrm{m}$ longitudinal sections cut throughout each block. Sets of consecutive sections, comprising the lesioned area, were used for immunostainning.

Behavior analysis. The behavioral analysis was performed by experimenters blinded to the treatment for 1 week before and for 5 weeks after injury. In the tape removal test (Bradbury et al., 2002), adhesive tape $(1.5 \times 1 \mathrm{~cm})$ was placed on each forepaw separately and the time taken to sense (indicated by paw shake) and remove the tape (cutoff time point, 1 min) was scored. For locomotor tasks (Bradbury et al., 2002), rats were trained to cross a horizontal ladder $(18-100 \mathrm{~cm}$ with each rung $5 \mathrm{~cm}$ apart) and the number of forelimb foot slips (off the beam or below the plane of the ladder) was recorded. Confirmation of injury was obtained by the poor performance of all animals in tape removal and locomotor tasks the day after injury. Behavioral responses of treated and control groups were compared using one-way ANOVA followed by Tukey's post hoc test.

Immunohistochemistry and antibodies. Immunohistochemistry was performed as described previously (Goncalves et al., 2005) Neuronal cultures were washed with PBS for $1 \mathrm{~min}$, fixed in $4 \%$ PFA for $30 \mathrm{~min}$, washed 3 times for 5 min each in PBS- $0.02 \%$ Tween, and incubated in primary antibody in PBS $-0.02 \%$ Tween at $4^{\circ} \mathrm{C}$ overnight. Primary antibody was removed by washing 3 times for 5 min each in PBS- $0.02 \%$ Tween, incubated in the secondary antibody for $1 \mathrm{~h}$ at room temperature in PBS- $0.02 \%$ Tween, and then washed in PBS 3 times for $5 \mathrm{~min}$. The coverslips were then mounted using FluroSave reagent (Merck). For spinal cord tissue, sections were first dewaxed in xylene and 100\% IMS, heated in citric acid (10 mM, pH 6) until boiling, and then washed under a running tap for $5 \mathrm{~min}$ before incubation with primary Ab. Antibodies used were as follows: mouse monoclonal anti- $\beta$ III tubulin (Promega, 1:1000 for immunohistochemistry and 1:10,000 for Western blotting); chicken polyclonal anti-GFAP (Abcam, 1:300); rabbit polyclonal antiGFAP (DAKO, 1:2500); mouse monoclonal anti-GFAP (Sigma-Aldrich, 1:100); rabbit polyclonal anti-RAR $\beta$ (Santa Cruz Biotechnology, 1:100 for immunohistochemistry and 1:500 for Western blotting); goat polyclonal anti-CGRP (Abcam, 1:200); rabbit polyclonal anti-PTEN (Abcam, 1:50); rabbit polyclonal anti-VGLUT1 (Abcam, 1:4000); rabbit polyclonal anti-c-Fos (Abcam, 1:300); rat polyclonal anti-synaptophysin (Serotec, 1:200); rabbit polyclonal anti-PTEN (Y184, Abcam, 1:500 for Western blotting); mouse monoclonal anti-PTEN (26H9, Cell Signaling Technology, 1:1000 for Western blotting); rabbit polyclonal antiphospho-PTEN (Ser380, Cell Signaling Technology, 1:500 for immunohistochemistry or 1:1000 for Western blotting); mouse monoclonal NMDAR2B (S59-36, Abcam, 1:500); rabbit polyclonal anti-Lamin A (H102, Santa Cruz Biotechnology, 1:250); and mouse monoclonal anti- $\beta$ actin (AC-15, Sigma-Aldrich, 1:5000). Secondary antibodies for immunohistochemistry were Alexa Fluor 594 and Alexa Fluor 488 (1: 1000 , Invitrogen). DAPI was used to stain nuclei $(1 \mu \mathrm{g} / \mathrm{ml}$, SigmaAldrich). Secondary antibodies for Western blotting were Alexa Fluor 680 (1:5000, Invitrogen) and IR Dye 800CW (1:5000, Li-Cor Biosciences). Quantitative analysis of GFAP-positive cells at the lesion site was performed as described previously (Herrmann et al., 2010). In brief, GFAP-positive areas were quantified as the pixels of immunoreactivity above a threshold level per unit area. The threshold value was set to 
include GFAP-positive signal and to exclude background staining. Threshold values for a given section and stain remained the same throughout the study. The number of pixels was measured from the implantation site of the severed dorsal roots to a distance of $100 \mu \mathrm{m}$ away from the implantation site. For BDA quantification, positively labeled axons were measured by mean intensity of immunoreactivity that had crossed the DREZ and entered the gray matter extending $300 \mu \mathrm{m}$ inwards. For synaptophysin and VGLUT1 quantification in the dorsal horn (lamina I and II for synaptophysin and II and III for VGLUT1), again, the mean intensity values of immunoreactivity obtained from highmagnification $(63 \times$ objective $)$ images of spinal cord sections taken at the same levels were used. c-Fos quantification was done as described previously (Yip et al., 2006). At least 10 sections per rat were used for these quantifications.

Confocal microscopy. Multichannel fluorescence (DAPI-FITC-Texas Red filter set) images were captured using a Zeiss LSM 700 laser-scanning confocal microscope with a $63 \times$ oil-immersion Aprochromat objective. Settings for gain, aperture, contrast, and brightness were optimized initially and held constant throughout each study so that all sections were digitized under the same conditions of illumination. Channels were imaged sequentially to eliminate bleedthrough and multichannel image overlays were obtained using Adobe Photoshop 7.0. For time-lapse imaging analysis, cells were imaged every $15 \mathrm{~min}$ for $24 \mathrm{~h}$ at $37^{\circ} \mathrm{C}$ and $5 \%$ $\mathrm{CO}_{2}$. Axiovision software was used to collect information on pixel immunoreactivity used for quantitative purposes.

Light micrography. Specimens intended for plastic embedding were perfusion fixed with $2 \%$ glutaraldehyde and rinsed in PBS. After dehydration in a graded series of ethanol, these specimens were embedded in Durcopan (Fluka). After polymerization, the specimens were cut in 0.5$\mu \mathrm{m}$-thick sections on an LKB Ultrotome $\mathrm{V}$ and stained with toluidine blue. Images were obtained with a Nikon E600 microscope with a $60 \times$ oil-immersion plan-apo lens and a Nikon Digital Sight DS-U1 camera.

After examination in the light microscope, some specimens were selected for electron microscopy. Ultrathin sections were prepared with a diamond knife and the LKB Ultrotome and collected on 400 mesh grids. After contrasting with a saturated solution of uranyl acetate and a $0.08 \mathrm{M}$ lead citrate solution, the sections were examined in an FEI Morgagni transmission electron microscope.

Diffusion MRI tractography. Specimens for ex vivo MRI were fixed in paraformaldehyde and transferred to Fombin in a plastic syringe and mounted in a $16 \mathrm{~mm}$ coil (Rapid Biomedical) in a 9.4 T MRI system (Agilent Technologies). Diffusion-weighted data were recorded overnight using a spin echo sequence $(\mathrm{TR}=4 \mathrm{~s}, \mathrm{TE}=18.17 \mathrm{~ms}, \mathrm{~b}=1250$ $\mathrm{s} / \mathrm{mm}^{2}, 70$ contiguous slices of $0.15 \mathrm{~mm}$ thickness, matrix $=192 \times 192$ $\mathrm{FOV}=19.2 \times 19.2 \mathrm{~mm}^{2}$, ucl42 scheme, zero-filled to $256 \times 256$ before reconstruction). Tractography DSI studio DTI reconstruction was performed using the following tracking parameters: termination index FA, threshold, $90^{\circ}$ angular threshold, step size 0.05 , subvoxel seed position, trilinear direction interpolation, and the streamline (Euler) tracking algorithm.

Primary neuronal cell cultures. Mouse primary cortical neurons were prepared as described previously (Goncalves et al., 2013). Cells were plated onto $10 \mu \mathrm{g} / \mathrm{ml}$ poly-D-lysine-coated $100 \mathrm{~mm}$ dishes at a density of $6 \times 10^{6}$ cells/dish. Cells were cultured in neurobasal medium (Invitrogen) containing 2\% (v/v) B27 serum-free supplement, 2 mm L-glutamine, 1.5\% glucose, penicillin $(100 \mathrm{units} / \mathrm{ml})$, and streptomycin $(100 \mu \mathrm{g} / \mathrm{ml})$ at $37^{\circ} \mathrm{C}$ in a humidified atmosphere of $5 \% \mathrm{CO}_{2} / 95 \%$ air. Cultures were used after 24 or $72 \mathrm{~h}$ and were $>98 \%$ neurons judged by $\beta$ III-tubulin staining. Treatments were as follows: mouse primary cortical cultures [0 $\mathrm{d}$ in vitro (DIV)] were treated with $0.1 \mu \mathrm{M}$ CD2019 for $72 \mathrm{~h}$ as indicated. For identification of PTENtransfected neurons, cells were visualized with CellTracker Red CMPTX (Life Technologies), which was added according to the manufacturer's instructions at the same time as treatments and placed in an incubator attached to a confocal microscope as described above. Pictures were taken on live cells every $30 \mathrm{~min}$ for $90 \mathrm{~min}$. Culture conditions were three wells per treatment performed three times.

Transfection of cortical neurons. Cortical neurons were plated at $1 \times$ $10^{6}$ cells/well in six-well plates. Separate solutions of cDNA in OptiMEM (Life Technologies) and Lipofectamine 2000 transfection reagent (Life
Technologies) in OptiMEM were prepared at a ratio of 1:1.9 ( $\mu \mathrm{g}$ DNA: $\mu 1$ Lipofectamine) and allowed to stand for $5 \mathrm{~min}$. They were then mixed and incubated at room temperature for $20 \mathrm{~min}$ before addition to the cells in a total volume of $2 \mathrm{ml}$. After $5 \mathrm{~h}, 1.5 \mathrm{ml}$ of medium was removed from each well and replaced with fresh $1.5 \mathrm{ml}$ aliquots of neurobasal medium containing L-glutamine, glucose, penicillin, streptomycin, and B27 supplement (all from Life Technologies). Human PTEN expression constructs was obtained from OriGene Technologies.

Mouse cortical neurons (10 DIV) were transfected with PTEN siRNA (SR413443) using siTRAN (1.0 transfection reagent; OriGene), according to the manufacturer's protocol. In brief, $1.5 \times 10^{-12} \mathrm{M}$ siRNA was added to each well and incubated for $72 \mathrm{~h}$. Knock-down of PTEN was confirmed by Western blotting in cells and in isolated exosomes. As a control, scrambled siRNA was added to wells (universal scrambled negative control siRNA duplex). These cells were then treated with either CD2019 $(0.1 \mu \mathrm{M})$ or with vehicle for $72 \mathrm{~h}$ and PTEN levels were measured in both exosomes and cell lysates, as described previously. Exosomes from similar preparations of transfected mouse cortical neurons treated either with CD2019 $(0.1 \mu \mathrm{M})$ or with vehicle for $72 \mathrm{~h}$ were isolated and used to treat astrocytes in culture to measure proliferation.

Neurite outgrowth assay. Exosomes isolated from CD2019- $(0.1 \mu \mathrm{M})$ and vehicle-treated astrocyte primary cultures were added to primary cortical neuron cultures in a one-to-one ratio (the quantity of exosomes isolated from one well of astrocytes was used to treat one well of neurons). After $72 \mathrm{~h}$, the cells were fixed in $4 \% \mathrm{PFA}$, permeabilized with $0.1 \%$ Triton X-100 for $4 \mathrm{~min}$, and stained with Alexa Fluor 488 Phalloidin (Invitrogen, 1:40). Images were captured as described before (in the "Confocal microscopy" section). Three fields capturing a minimum of 10 neurons were taken per coverslip in at least three coverslips for each culture condition. The images were processed using ImageJ software. The length of the longest neurite in each neuron was measured. Statistical analysis was done with one-way ANOVA followed by Tukey's test.

Astrocyte cultures. Primary mixed glial cultures were prepared as described previously (Goncalves et al., 2013) using a modified protocol. Briefly, mixed glial cultures were obtained from the cortices of postnatal (P) mice (P5-P8). Cultures were maintained at $37^{\circ} \mathrm{C}\left(5 \% \mathrm{CO}_{2} / 95 \% \mathrm{O}_{2}\right)$ in medium containing 15\% fetal bovine serum (FBS) (Invitrogen) and $1 \%$ penicillin-streptomycin (Sigma-Aldrich) for 10-14 d. Microglial cells were then harvested by forceful shaking for $1 \mathrm{~min}$ by hand. After harvesting of microglia was done every $3 \mathrm{~d}$ for $9 \mathrm{~d}, 0.25 \%$ trypsin EDTA was added to each flask for $15 \mathrm{~min}$. Cells were then centrifuged at 2000 rpm for $10 \mathrm{~min}$ and plated on poly-D-lysine-coated coverslips (24-well plates) or 96-well plates in 5\% FBS.

Cell fractionation. All centrifuge steps were performed at $4^{\circ} \mathrm{C}$. Mouse cortical neurons were harvested in hypotonic buffer (10 mM HEPES, $\mathrm{pH}$ 7.5, $10 \mathrm{~mm} \mathrm{KCl}, 3 \mathrm{~mm} \mathrm{MgCl}$, 1 mm NA $\mathrm{VO}_{4}, 1 \mathrm{~mm} \mathrm{NaF}$, and EDTA-free protease inhibitor mixture; Roche). Cell lysates were left on ice for $20 \mathrm{~min}$ and then passed through a $25 \mathrm{Ga}$ needle $10 \times$ and then centrifuged at $720 \times g$ for $5 \mathrm{~min}$. The supernatant (SN1) was collected and the pellet (P1, nuclear pellet) was washed once in hypotonic buffer, centrifuged again at $720 \times g$ for $5 \mathrm{~min}$, and then resuspended in $2 \times$ Laemmli sample buffer. SN1 was further centrifuged at $10,000 \times g$ and the supernatant (SN2) was collected and the pellet (P2, mitochondrial pellet) was discarded. SN2 was then centrifuged at $90,000 \times g$ for $1 \mathrm{~h}$. The supernatant (SN3, cytosolic fraction) was collected and assayed for protein concentration. The pellet (P3, membrane fraction) was resuspended in Triton buffer (10 mm Tris, pH 7.4, 500 mм NaCl, 1 mм EDTA, 1\% Triton X-100, $1 \mathrm{~mm} \mathrm{NA}_{3} \mathrm{VO}_{4}, 1 \mathrm{~mm} \mathrm{NaF}$, and EDTA-free protease inhibitor mixture; Roche). After resuspension, protein lysates were centrifuged for $30 \mathrm{~min}$ at $16,000 \times g$ and the supernatant (SN4, membrane proteins) was collected. Whole-cell lysates were made by incubating cell pellets directly in RIPA buffer (25 mm Tris, pH 7.4, $150 \mathrm{~mm} \mathrm{NaCl}, 1 \% \mathrm{NP}-40,1 \%$ sodium deoxycholate, $1 \mathrm{~mm} \mathrm{NaF}, 1 \mathrm{~mm} \mathrm{Na} \mathrm{VO}_{4}, 0.1 \%$ SDS, $1 \mathrm{~mm}$ PMSF, $10 \mathrm{~mm}$ EDTA, supplemented by EDTA-free protease inhibitor mixture). Protein concentrations were determined with the BCA Protein Assay (Pierce).

Western blotting. Proteins were separated by SDS-PAGE on 10\% (w/v) polyacrylamide gels and then transferred to a $0.45 \mu \mathrm{m}$ pore size nitrocellulose membrane (BA85; Schleicher and Schuell) using a Trans-Blot SD Semi-Dry Transfer Cell (Bio-Rad Laboratories) Nitrocellulose mem- 
A

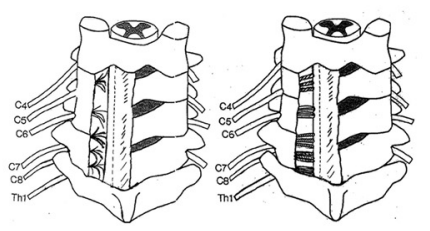

E

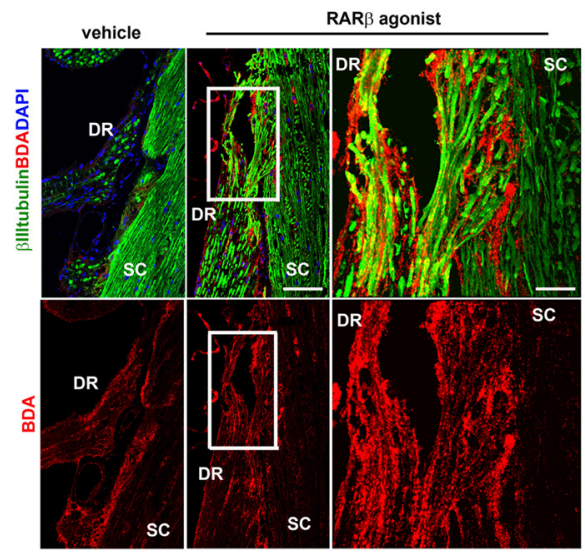

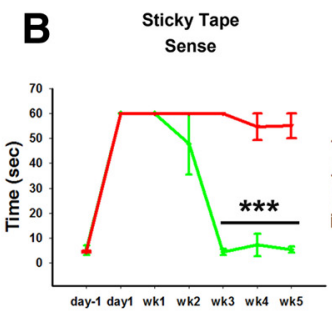

$\mathbf{F}$

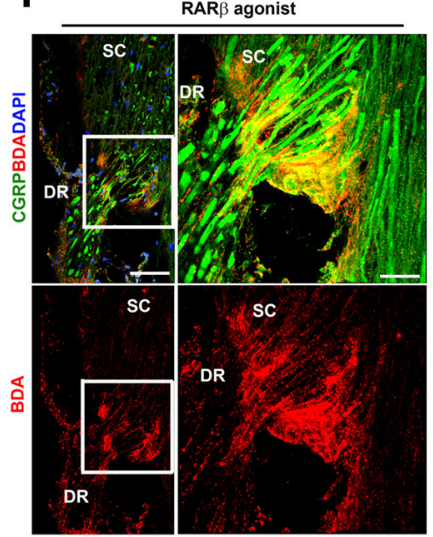

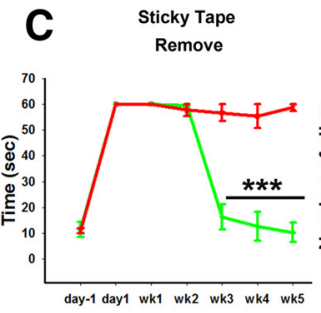

G

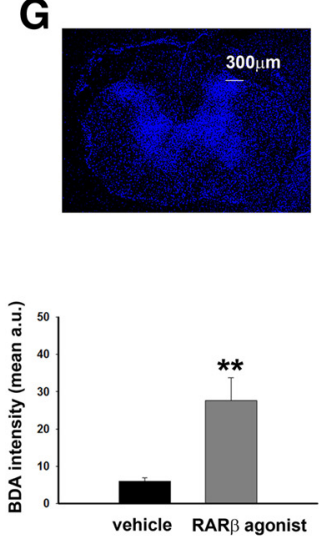

D Horizontal ladder

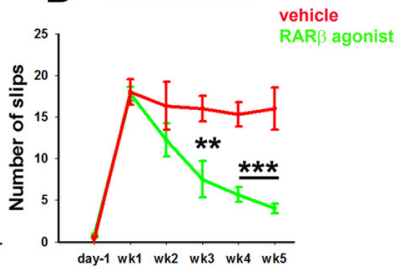

H

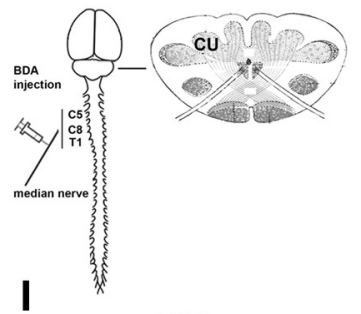

BDA

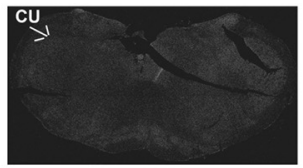

Figure 1. The RAR $\beta$ agonist promotes functional recovery and axonal regeneration. $A$, Schematic of the injury performed, in which C5-T1 were cut and grafted back into the SC Rats were assessed in behavioral tasks before and for 5 weeks after avulsion. $B, C$, In a tape removal task, the time taken to sense and remove the tape by the lesioned forelimbs was measured and significantly lower latencies were observed with the injured forelimbs of RAR $\beta$ agonist ( $1 \mathrm{mg} / \mathrm{kg}$, i.p., every other day)-treated rats compared with vehicle-treated ones, from week 3 of treatment. $D$, In locomotor tasks, the number of foot slips in a horizontal ladder made by the injured forelimb of RAR $\beta$-agonist-treated rats was markedly lower than that of vehicle-treated rats from week three. Data represent mean \pm SEM. ${ }^{* *} p \leq 0.005 ;{ }^{* * *} p \leq 0.001$, one-way ANOVA followed by Tukey's post hoc test. $\boldsymbol{E}, \boldsymbol{F}$, BDA-labeled axons double stained with either $\beta$ III tubulin $(\boldsymbol{E})$ or $(\mathrm{GRP}(\boldsymbol{F})$ can be seen along the dorsal root and crossing the DREZ in RAR $\beta$-agonist-treated rats, but not in vehicle-treated ones. Scale bars: left, $100 \mu \mathrm{m}$; $\boldsymbol{E}$, right insets, $50 \mu \mathrm{m}$, left insets $80 \mu \mathrm{m} ; \boldsymbol{F}$, insets, $80 \mu \mathrm{m}$. G, Quantification of BDA-labeled axons that cross the DREZ into the dorsal horn up to $300 \mu \mathrm{m}$ into the gray matter. $\boldsymbol{H}$, Schematic representation of the median nerve (BDA injection site) ascending tracts into the brainstem in the cuneate nucleus (CN). $I$, Absence of BDA in CN nerve tracts in an RAR $\beta$-treated rat. Results are mean \pm SEM. ${ }^{* *} p \leq 0.005$, Student's $t$ test.

branes were incubated in blocking solution consisting of $5 \%(\mathrm{w} / \mathrm{v})$ skimmed milk powder in PBS-Tween-20 (0.1\%, w/v) (PBS-T) for $1 \mathrm{~h}$ at room temperature, followed by incubation with the appropriate primary antibody diluted in blocking solution overnight at $4^{\circ} \mathrm{C}$. Membranes were washed in PBS-T and then incubated with species-specific secondary antibodies in blocking solution for $1 \mathrm{~h}$ at room temperature in the dark, after which time the membranes were washed as above. Protein levels were corrected for loading differences by normalizing against $\beta$-actin levels. Proteins were detected by scanning at 700 and $800 \mathrm{~nm}$ using the Odyssey detection system (LI-COR Biosciences).

Exosomes. Exosomes were prepared from conditioned media of cortical neurons treated with the RAR $\beta$ agonist CD2019 (0.1 $\mu \mathrm{M})$ or dimethylsulfoxide vehicle using Total Exosome Isolation (TEI) reagent (Invitrogen) in accordance with the manufacturer's instructions. Conditioned media were centrifuged at $2000 \times g$ for $30 \mathrm{~min}$ at $4^{\circ} \mathrm{C}$ to remove cells and cell debris and the resulting supernatants were mixed with 0.5 volumes of TEI reagent, incubated overnight at $4^{\circ} \mathrm{C}$ and centrifuged at $10,000 \times g$ at $4^{\circ} \mathrm{C}$ for $1 \mathrm{~h}$. Exosome pellets were twice washed by resuspension in ice-cold PBS followed by centrifugation at $100,000 \times g$ at $4^{\circ} \mathrm{C}$ for $1 \mathrm{~h}$. For Western blots, pellets were lysed in a buffer containing $25 \mathrm{~mm}$ Tris-HCl, pH 7.4, 150 mм NaCl 1\% Triton X-100, 1 mм EDTA, 10\% (v/v) glycerol, $1 \mathrm{~mm}$ phenylmethylsulfonylfluoride, $1 \mathrm{~mm}$ sodium fluoride, and EDTA-free protease inhibitor mixture (Roche). Lysates were triturated 10 times through $29 \mathrm{Ga}$ needles. As a control for exosomal proteins, CD2019- and vehicle-treated cortical neurons were washed with PBS, collected, and lysed as above. All lysates were centrifuged for 15 $\min$ at $21,000 \times g$ at $4^{\circ} \mathrm{C}$ and the protein concentration in the supernatants was determined with Pierce BCA Protein Assay reagents. Unused intact exosomes were stored at $-80^{\circ} \mathrm{C}$ as PBS suspensions. For exosome injections at the implantation site of severed dorsal roots, a pool of exosomes isolated from $75 \mathrm{~cm}^{2}$ Thermo Scientific cell-culture-treated flasks with filter caps of $99 \%$ confluent mouse cortical neurons that had been treated with vehicle or CD2019 as before was used. A single unilat- eral injection of $10 \mu \mathrm{l}$ of exosome stock was made manually using a $20 \mu \mathrm{l}$ Hamilton syringe at $0.5 \mu \mathrm{l} \mathrm{min}{ }^{-1}$ and the needle was left in place for 5 min to limit exosome diffusion through the needle tract.

Cell proliferation assay. Astrocytes plated in 96 -well plates at $60 \%$ confluency were treated with either CD2019 $(0.1 \mu \mathrm{M})$ or dimethylsulfoxide $\left(\begin{array}{ll}1 & \mu l\end{array}\right)$ or with exosomes isolated from the conditioned medium of CD2019 $(0.1 \mu \mathrm{M})$-treated or dimethylsulfoxide-treated cortical neurons. In a similar setup, astrocytes were treated with exosomes isolated from siRNA (scrambled or PTEN)-transfected cortical neurons cultured with CD2019. After overnight incubation at $37^{\circ} \mathrm{C}$ in $5 \% \mathrm{CO}_{2}$, cell proliferation was determined by the CellTiter 96 Non-Radioactive Cell Proliferation Assay kit (Promega) in accordance with the manufacturer's instructions. Aliquots of $15 \mu \mathrm{l}$ of dye were added to the cells for $4 \mathrm{~h}$. Solubilization/stop solution $(100 \mu \mathrm{l})$ was added overnight and the absorbance at $570 \mathrm{~nm}$ was read using a plate reader.

Data analysis. Data were analyzed using Student's $t$ test with one-way ANOVA followed by Tukey's or Fisher's test, using SigmaStat software (SPSS). Comparisons were made between appropriate groups and differences were considered statistically significant at the level of $p<0.05$. Results are given as mean \pm SE and $p$ values are provided as summary statistics.

\section{Results}

\section{$\operatorname{RAR} \beta$ agonist induces functional recovery after cervical dorsal root injury}

We modeled in the rat a human avulsion injury in which the sensory dorsal roots were cut flush with the SC surface leading to the left forelimb at C5-C8 and T1 (Fig. 1A). The cut ends of the dorsal roots were subsequently introduced through slits in the pia mater and positioned superficially in the SC adjacent to where they had been cut (Fig. 1A). The motor roots were left intact because they have the capacity for some regeneration after this type of injury (Cullheim et 
al., 1989). Three days after surgery, rats were injected intraperitoneally with an RAR $\beta$ agonist ( $1 \mathrm{mg} / \mathrm{kg}$ ) every other day for a period of 4 weeks. All animals were trained preoperatively for 2 weeks in sensory and locomotor tests, which consisted of the sensing and removing of sticky tape from the paw and the number of foot slips made walking across a horizontal ladder. Performances were recorded the day before surgery, the day after surgery, and every following week until postoperative week five (inclusive). C5-T1 rhizotomy (Fig. 1A) produced an immediate, complete functional loss in the pertinent forelimb. Although there was no recovery in the vehicle-treated animals, there was a significant improvement in the agonist-treated ones from the third week of treatment (Fig. $1 B-D$ ).

\section{RAR $\beta$ agonist induces axonal regeneration and reduced glial scar size in avulsed rats}

To confirm that the functional recovery was the result of true axonal regeneration rather than sprouting and/or compensation from spared axons, the median nerve was injected with the tracer BDA 3 weeks before the rats were killed at week six. Spinal cords with DRGs and roots attached were dissected out for histology analysis. To observe regeneration only in sensory roots C6-C8 and avoid including misleading sprouting from intact roots adjacent to the injury site, we used calcitonin gene-related peptide (CGRP), which is widely expressed in sensory neurons and is upregulated in regenerating axons ( $\mathrm{Li}$ et al., 2004), along with BDA double labeling. No BDA-labeled axons were found to have crossed the DREZ in vehicle-treated animals, whereas robust regeneration could be seen in RAR $\beta$-agonist-treated rats (Fig. $1 E, F)$. In these, a significant number of sensory axons, colabeled with $\beta$ III tubulin and CGRP, could be seen projected from the dorsal root into the dorsal horn (Fig. 1E,F). Quantification of BDA-labeled axons $300 \mu \mathrm{m}$ into the dorsal horn confirmed the significant degree of regeneration in agonist compared with vehicle-treated animals (Fig. $1 G$ ). To rule out sprouting from spared axons, we then looked at BDA-labeled nerve tracts in the cuneate nucleus in RAR $\beta$-agonist-treated rats. The absence of staining in these ascending nerve tracts in the brainstem (Fig. $1 H, I)$ confirmed a complete lesion and that the BDA-positive axons present in the dorsal horn represent regenerating axons.

We next sought further confirmation of axonal regeneration by examining various markers of regeneration and synaptic connectivity. All rats were subject to noxious heat stimuli in their forelimbs, which induces c-Fos activation in postsynaptic neurons in the superficial laminae of the dorsal horn (Hunt et al., 1987). We found that immunoreactivity of c-Fos was greater in laminae I and II of the dorsal horn in RAR $\beta$-agonist-treated rats than in vehicle-treated rats (Fig. $2 A, B$ ). Next, we looked at CGRP in conjunction with synaptophysin, a presynaptic terminal marker. CGRP nociceptors restrict their terminals to lamina I and the outer aspect of lamina II and the reestablishment of synaptic connections to the most appropriate target region is paramount to achieving functional recovery (Sanes and Yamagata, 1999). Analysis of synapse formation by double labeling CGRPpositive axons for synaptophysin demonstrated extensive ectopic synapses in the outer laminae of the dorsal horn in SC segments connected to the injured dorsal roots, but only in RAR $\beta$-agonisttreated animals (Fig. 2C,D). Furthermore, motoneurons in the ventral horn of treated animals exhibited higher levels of RAR $\beta$ and were notably more densely surrounded by synaptophysin vesicles (Fig. $2 E$ ). This may suggest that $R A R \beta$ signaling might either restore or preserve synaptic connections between dorsal and ventral horn neurons. We next analyzed the recovery of mechanoceptive and proprioceptive synapses, which are lost as a consequence of nerve injury (Alvarez et al., 2011). We found that VGLUT1, which is the principal excitatory neurotransporter from primary afferents onto spinal neurons, was strongly expressed in RAR $\beta$-agonist-treated rats, but not in vehicle-treated ones (Fig. $2 F-H$ ).

To further assess the presence of regenerated axons in the SC, plastic-embedded specimens from $\operatorname{RAR} \beta$-treated animals of the SC and implanted dorsal roots were cut longitudinally with the implanted root. Light micrographs showed that, at the site of implantation, bundles of myelinated fibers could be followed from the root into the SC (Fig. $3 A, B$ ). The nerve fibers in this region were seen to be typically separated from each other by fibrous astrocytic processes. Several histological features corroborate that true regeneration from the root into the SC has occurred: the finding of typical transitional nodes of Ranvier where the myelinated fiber changes from a peripheral to a central type of myelin and the presence of juxtaposed astrocytic nucleus (Berthold et al., 1993; Berthold and Carlstedt, 1977a, 1977b; Fig. $3 A, B)$. Importantly, the surrounding tissue lacks the cellular infiltrate of a glial scar, whichis typically formed by a complex system of interacting cell types (Cregg et al., 2014).

Electron microscopy also showed PNS to CNS transition of myelinated fibers. In ultrathin sections adjacent to the semithin sections for light microscopy, most of the proximal PNS type of paranode covered by basement menbrane occurred between longittudinally arranged astrocytic processes (Fig. 3C). Because glial scar formation inhibits axon regrowth (Silver and Miller, 2004) but a contained number of astroglia are required for essential functions of tissue repair and neuroprotection (Bush et al., 1999; Faulkner et al., 2004), we looked closer at the GFAP population at and beyond the DREZ in each animal group. Whereas BDAlabeled axons stopped at the glial barrier formed at the DREZ in vehicle-treated rats, they extended all the way through into the dorsal horn in $\operatorname{RAR} \beta$-agonist-treated rats, which exhibited a considerably smaller population of GFAP-positive cells in the dorsal root adjacent to the implantation site (Fig. $4 A, C$ ). Highresolution imaging showed that the morphology and density of the glial cells was remarkably different, with a glial scar formed by hypertrophic astrocytes with mesh-like entangled processes in vehicle-treated rats, whereas in RAR $\beta$-agonist-treated rats, elongated tunnel-like astrocytes were seen bridging the PNS and CNS (Fig. 4B). The phenotypes of the glia observed in each animal group have been well characterized and ascribed to regeneration nonpermissive scar and permissive glia axonal support network, respectively (Cregg et al., 2014).

Additional evidence for axonal regeneration from the severed dorsal roots into the SC in $\mathrm{RAR} \beta$-agonist-treated animals was obtained using a 9.4 T MRI scanner. The vector pictures of voxels representative of water diffusion seen in the animal treated with an RAR $\beta$ agonist showed a predominant orientation indicative of anatomical structures that could be nerve fibers and/or astrocytic processes that are aligned through the white matter and the replant (Fig. $5 A, C$ ), as suggested in the histology.

In contrast, it was not possible to find any signs of connectivity between the white matter and the replant in the animal treated with the control vehicle (Fig. $5 B, D$ ). Together with the histology and functional data, these results, although not conclusive per se, provide support for axonal regeneration and connectivity.

\section{RAR $\beta$ signaling modulates axonal regeneration via PTEN phosphorylation}

The notable modulation of glial cell activity and axonal regeneration induced by RAR $\beta$ agonist treatment could only be ex- 


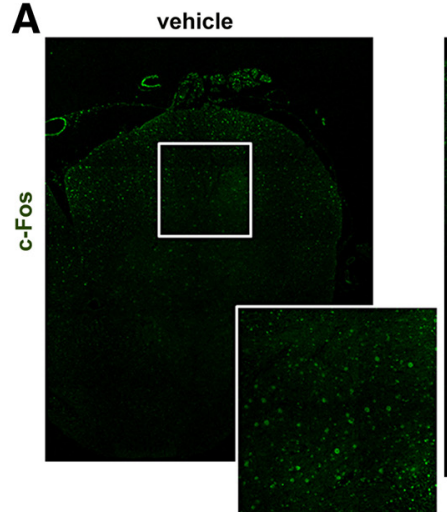

C

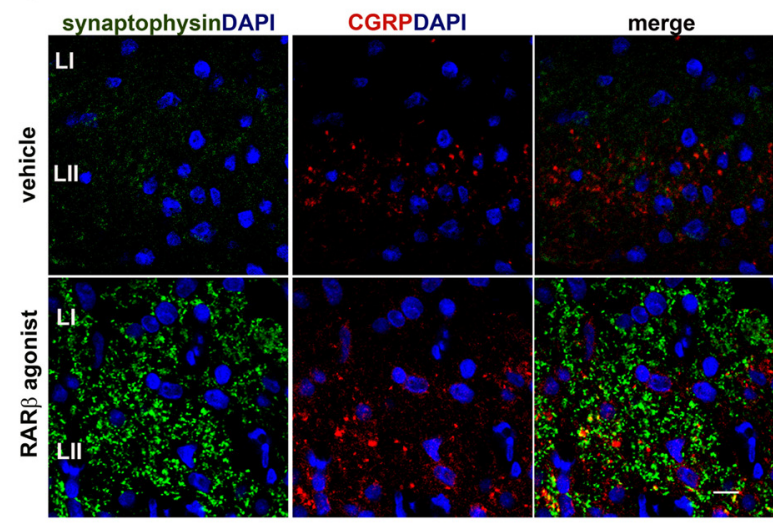

E
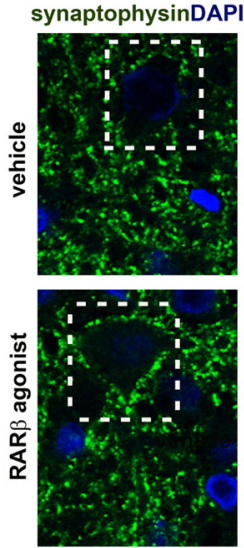

G

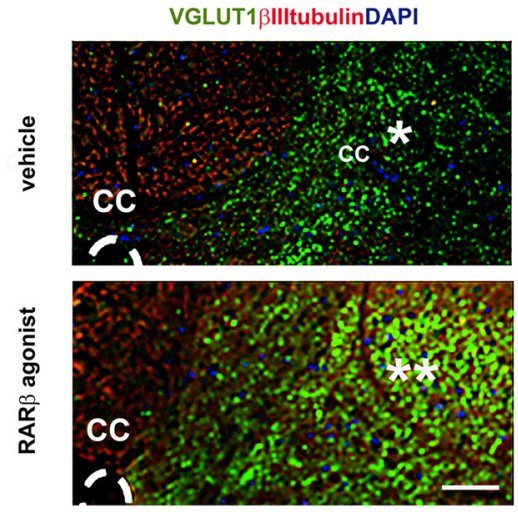

RAR $\beta$ agonist

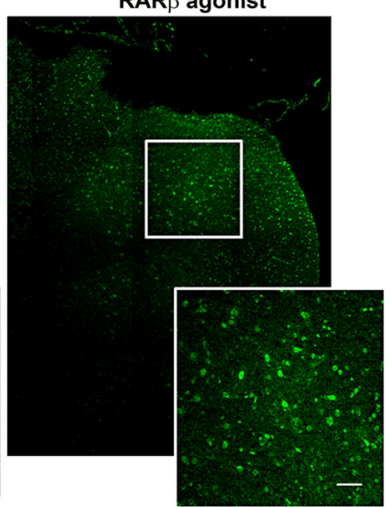

RARBDAPI
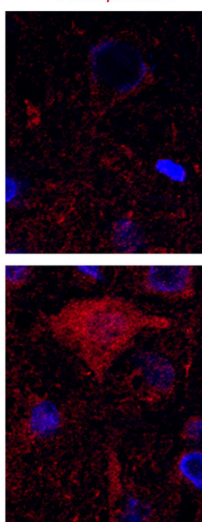

VGLT1ßIIItubulinDAP
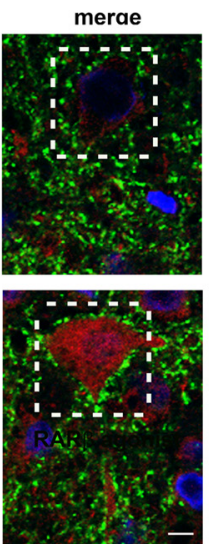

B

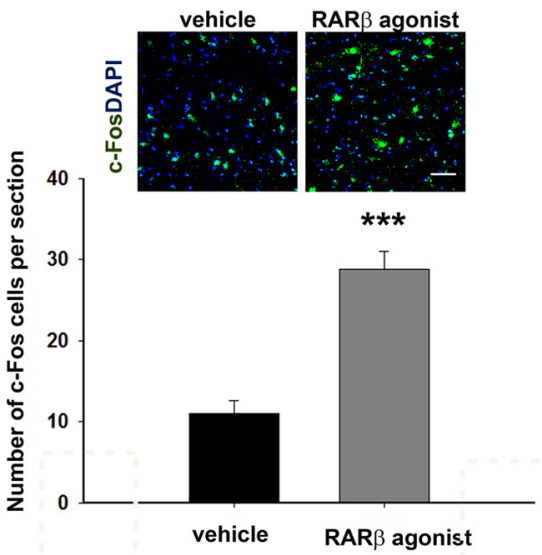

D

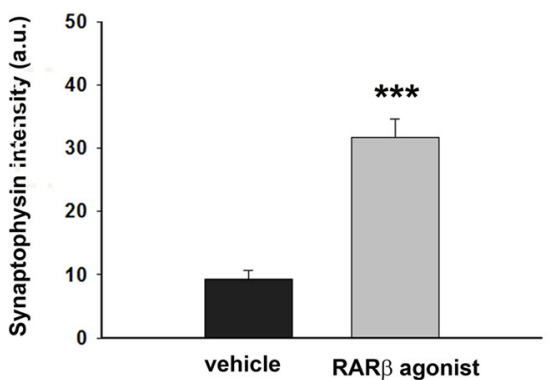

H

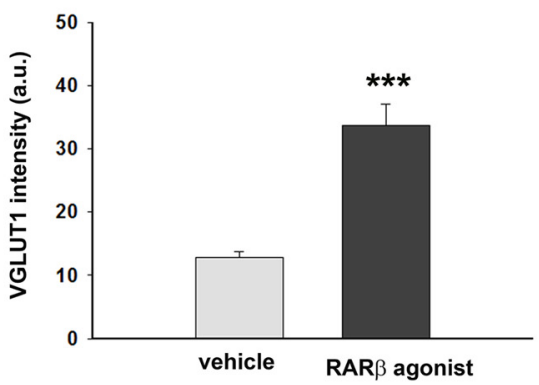

Figure 2. RAR $\beta$ agonist induces peripheral afferent stimulation and activation of postsynapticneurons in the spinal cord. $A, B$, After noxious heat stimulation, a significantly higher number of c-Fos-immunoreactive nuclei were detected in the SC of lesioned RAR $\beta$-agonist-treated rats compared with vehicle-treated ones. Scale bars: $\boldsymbol{A}, 50 \mu \mathrm{m} ; \boldsymbol{B}, 75 \mu \mathrm{m}$. C, $\boldsymbol{D}$, Expression of presynaptic marker synaptophysin and CGRP in the dorsal horn laminae I and II (LI and LII) is much higher in RAR $\beta$-agonist-treated rats then in vehicle-ones. Scale bar, $20 \mu \mathrm{m}$. $\boldsymbol{E}$, High levels of RAR $\beta$ and synaptophysin are seen in the ventral horn neurons (dotted lines) in RAR $\beta$-agonist-treated rats but not in vehicle ones. Scale bar, $10 \mu \mathrm{m}$. $\boldsymbol{F}$ - $\boldsymbol{H}$, Higher expression of VGLUT1 in neurons in the dorsal horn (LII and LIII) in RAR $\beta$-agonist-treated rats than in vehicle-treated rats. Scale bars: $\boldsymbol{F}, 50 \mu \mathrm{m} ; \boldsymbol{G}, 100 \mu \mathrm{m}$. CC, Central canal. Results are mean \pm SEM. ${ }^{* * *} p \leq 0.001$, Student's $t$ test $(\boldsymbol{B}, \boldsymbol{D}, \boldsymbol{H})$. 

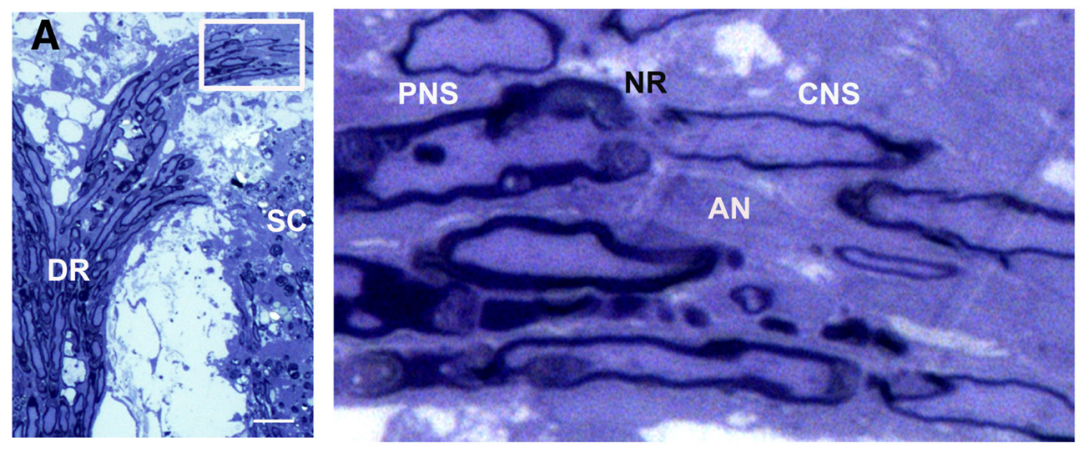

B
PNS

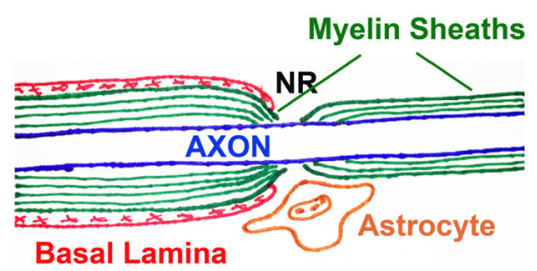

Basal Lamina

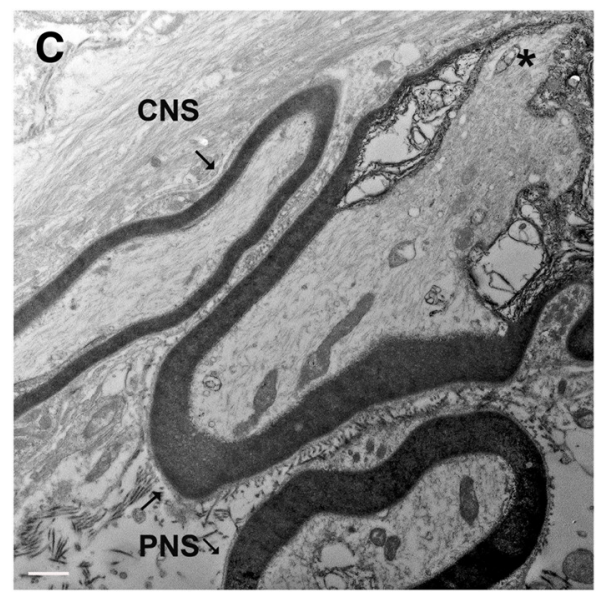

Figure 3. Regeneration from the PNS into the CNS. Light micrograph (LM) of longitudinal section through dorsal root implantation into a pocket of $\mathrm{SC}$ in plastic-embedded specimen. There are signs of previous trauma, such as lack of a glia limitans, vacuoles, and posttraumatic edema. Note also the lack of a glia scar formation at the site of SC implantation. Inset shows a bundle of myelinated fibers crossing from PNS to CNS. The most proximal peripheral paranodes are surrounded by astrocytic processes. A transitional PNS-CNS node of Ranvier (NR) is seen with atypical adjacent astrocytic nucleus (AN). Scale bar, $100 \mu \mathrm{m} . \boldsymbol{B}$ shows a diagram of $\boldsymbol{A}$. $\boldsymbol{C}$, EM micrograph of ultrathin section from the same region as shown in LM $(\boldsymbol{A})$. The most proximal PNS type of paranode (indicated by the asterisk) with a typical Schwan cell basement membrane. The paranode, when in this region, is typically surrounded by fibrous astrocytic processes that have arranged themselves in "tunnel"-like formations. The basement membrane seen at the surface of this tunnel is a reflection of the Schwann cell basement membrane of the paranode, which at the composite PNS-CNS node of Ranvier, leaves the pertinent nerve fiber and follows the outline of the glia tunnel. The arrow indicates a basal lamina. Scale bar, $500 \mathrm{~nm}$.

plained if $\operatorname{RAR} \beta$ signaling acts on a key pathway determining regeneration. PTEN is a major contributor to adult regenerative failure in the CNS, in which membrane-bound PTEN inhibits PI3K (Maehama and Dixon, 1998; Carracedo and Pandolfi, 2008; Park et al., 2010).

We showed previously that RAR $\beta$ signaling stimulates the PI3K pathway in cultured cerebella neurons via a nongenomic mechanism, resulting in neurite outgrowth (Agudo et al., 2010). Here, we postulated that activation of RAR $\beta$ signaling after nerve injury could promote regeneration via inactivation of PTEN. To confirm this, we cultured cortical neurons and stimulated them for $72 \mathrm{~h}$ with an $\operatorname{RAR} \beta$ agonist $(0.1 \mu \mathrm{M})$. This induced an increase in expression of $\mathrm{RAR} \beta$ in the cytoplasm and a decrease in the membrane compared with vehicle-treated cultures (Fig. 6A, $B$ ). Interestingly, this correlated with the levels of PTEN, which significantly increased in the cytoplasm and decreased in the membrane (Fig. 6A,B). We also found that, in RAR $\beta$-treated cultures, there was an increase in cytoplasmic phosphorylated PTEN (Fig. 6A,B), which represents an inactive form of the protein (Ross and Gericke, 2009). To confirm these data and to rule out a transcriptional upregulation of PTEN, we performed live-cell imaging using GFP-PTEN under control of a CMV promoter. This was transfected into neurons and the cytoplasm visualized by using a red fluorescent cell tracker. At time 0 ,
PTEN could be detected in the neurites and there was very little overlap with the cell tracker, suggesting that PTEN was located in the membranes (Fig. 6C). Next, we stimulated the cultures with $0.1 \mu \mathrm{M} \operatorname{RAR} \beta$ agonist. With increasing time $(0-60 \mathrm{~min})$, the PTEN became located in the cytoplasm, as shown by its overlap with the cell tracker and concomitant decrease in the neurites, and eventually the majority was found in the neuronal cell body (90 min) (Fig. 6C). We also found that $\operatorname{RAR} \beta$ agonist treatment resulted in increased nuclear PTEN, both total and phosphorylated. Together, these results show that RAR $\beta$ signaling modulates the cellular distribution of PTEN.

\section{RAR $\boldsymbol{\beta}$ signaling modulates neuron- glial cross talk via PTEN transfer in exosomes}

The data above suggest that an inactivation of PTEN is in part responsible for the axonal regeneration that we see in the injured neurons. Because we had found that the scar size was significantly reduced in agonist-treated compared with vehicle-treated animals (Fig. $4 D$ ), we inferred that RAR $\beta$ signaling might also affect scar formation. A possible mechanism of action would be to stop the formation of the scar either by a direct action of the agonist on the glial cells or an indirect mechanism via neuronal-glial signaling. Exosomes take part in intercellular communication (Chivet et al., 2013) and it has been shown recently that this also applies to axonal regeneration in the PNS (LopezVerrilli et al., 2013). Because PTEN regulates astrocyte proliferation (Fraser et al., 2004) and can reduce cell proliferation via exosome trafficking (Putz et al., 2012), we postulated that the RAR $\beta$ agonist might induce neuronal secretion of PTEN in exosomes that target astrocytes and hamper their proliferation. To test this, we extracted exosomes from culture media of neurons that had been treated with and without RAR $\beta$ agonist for $72 \mathrm{~h}$. PTEN content was measured in each pool of exosomes and we found that the ones treated with CD2019 were significantly enriched in PTEN (Fig. $7 A, B$ ). Next, we added exosomes to glial cultures and measured proliferation after $24 \mathrm{~h}$. In RAR $\beta$-agonist-treated cultures, there was little or no effect on proliferation compared with vehicletreated cultures (Fig. 7C). In exosome-treated cultures from nonCD2019-treated neurons, proliferation was comparable to that of vehicle-treated neurons. However, in cultures treated with exosomes from agonist-stimulated neurons, there was a dramatic decrease in astrocyte number (Fig. 7C). To confirm that this was indeed the result of neuronal-exosome-secreted PTEN that had been taken up by astrocytes, we repeated the experiment and immunostained the cultures for PTEN. We found that little or no PTEN could be detected in the astrocytes except for the ones cultured with exosomes from CD2019-treated neurons, in which there was a remarkable increase in PTEN expression (Fig. 7D). To verify that CD2019 did not affect endogenous PTEN in astrocytes directly via an intracellular or exosome-mediated effect, astrocyte cultures were treated with the $\operatorname{RAR} \beta$ agonist and exosomes were 

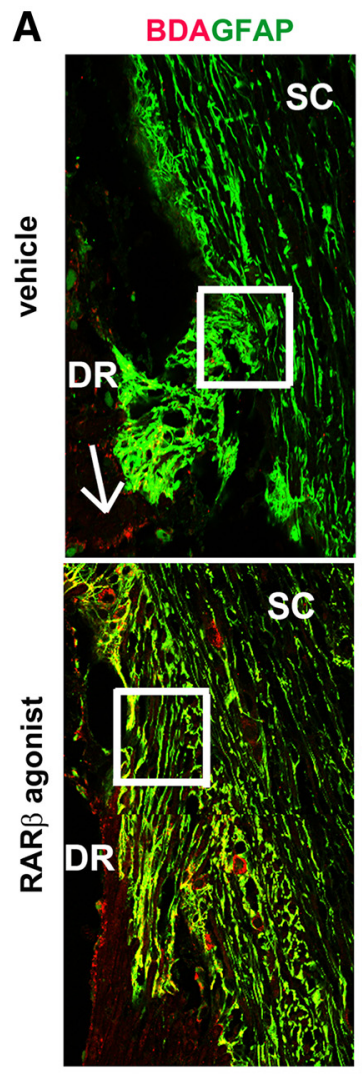

GFAP
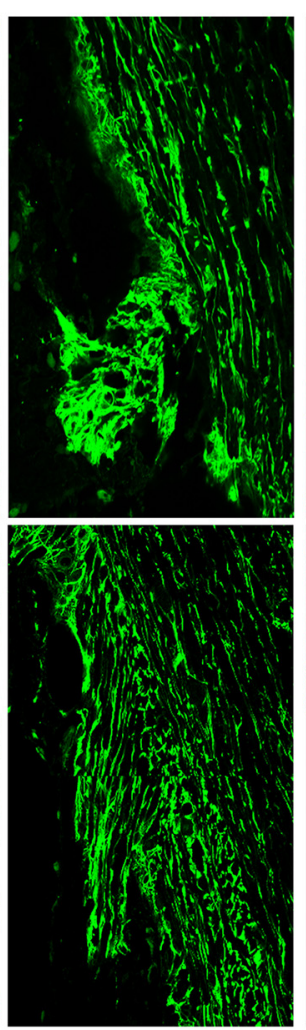

BDA
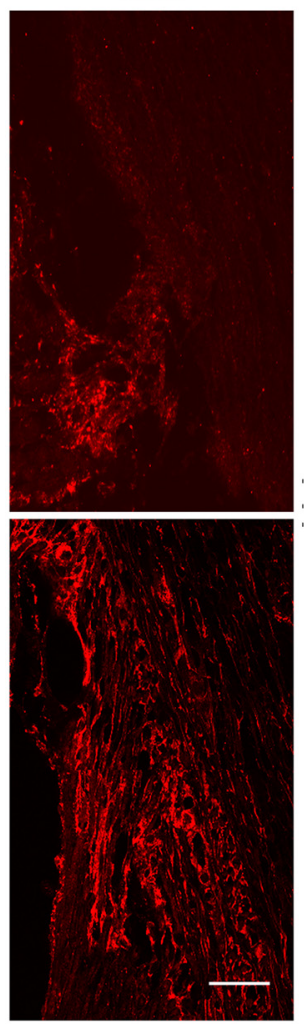

B
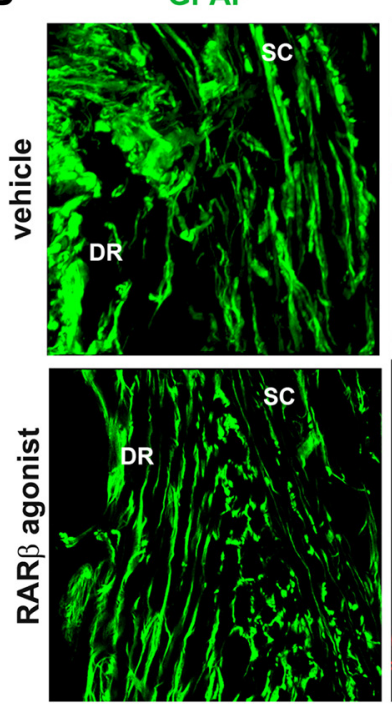

C

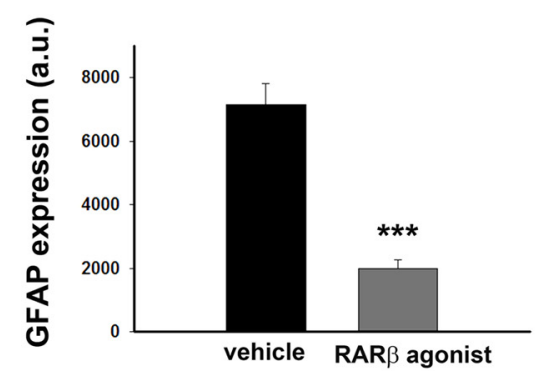

vehicle RAR $\beta$ agonist
BDA
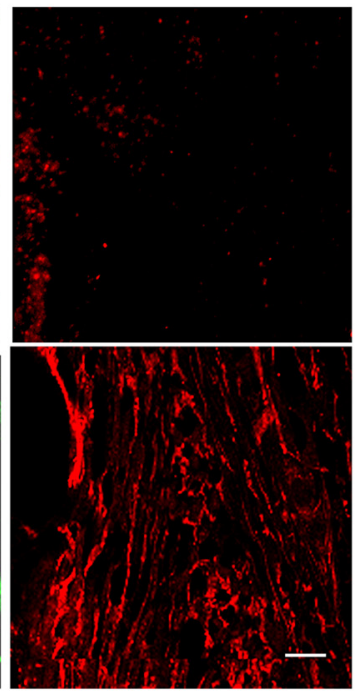

Figure 4. Absence of glial scar in RAR $\beta$-agonist-treated rats at the DREZ. A, Differences in astrocytes at the reimplantation site in vehicle- and RAR $\beta$-agonist-treated rats. Scale bar, $100 \mu \mathrm{m}$. $B$, High-resolution Z-stack images of insets shown in $\boldsymbol{A}$ in which differences in astrocyte morphology and density at the DREZ is clear between the two groups. Scale bar, $20 \mu \mathrm{m}$. C, Quantification of GFAP. Results are mean \pm SEM. ${ }^{* * *} p \leq 0.001$, Student's $t$ test.

extracted from the medium after $72 \mathrm{~h}$. Western blots of cell lysates showed no difference in PTEN nor phosphorylated PTEN expression compared with control cultures (Fig. $7 E, F$ ). Similarly, little or no PTEN was detected in the exosomes treated with or without the RAR $\beta$ agonist (data not shown). This suggests that it is a neuron-glial transfer of PTEN in exosomes that is the operative mechanism by which $\operatorname{RAR} \beta$ activation results in increased astrocytic PTEN.

To ascertain whether a similar mechanism occurs in vivo, we next looked at PTEN in astrocytes in the perilesioned area of avulsed rats that had been treated with either vehicle or the $\operatorname{RAR} \beta$ agonist. An increase of PTEN would be expected given the lack of glial scar sustained by the drug-treated rats. As anticipated, PTEN was highly expressed in astrocytes at the level of the site of injury in agonist-treated rats, but not in vehicle-treated rats (Fig. $7 G$ ).

Because exosomes carry several proteins, lipids, and RNA that can exert biological activity in the recipient cells (Raposo and
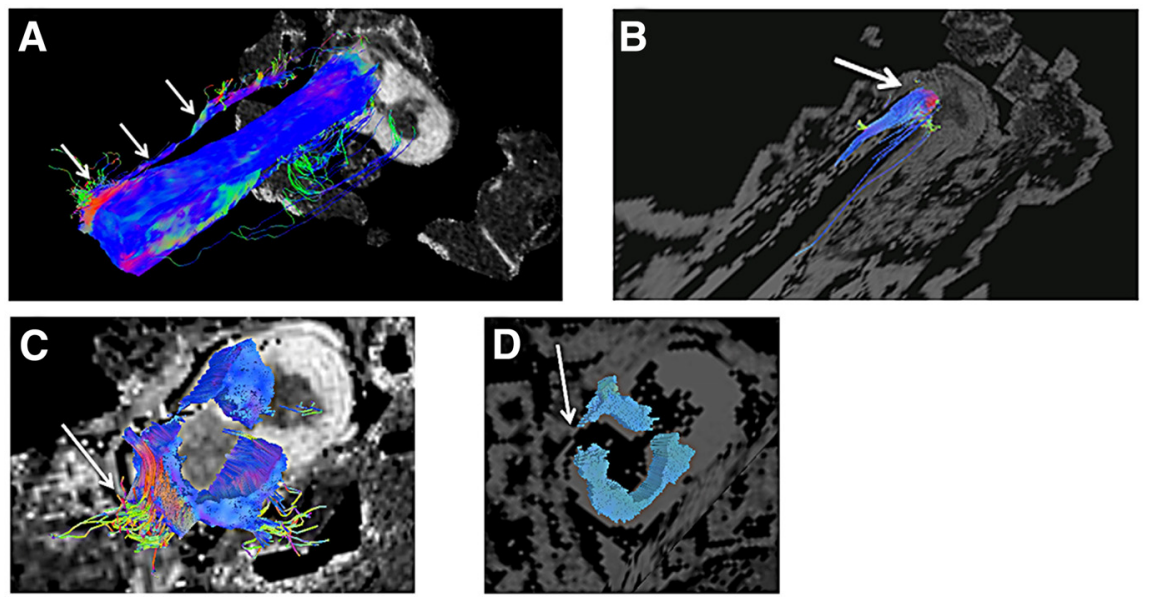

Figure 5. Fiber-tracking images of nerve roots in avulsed rats. The images $(\boldsymbol{A}-\boldsymbol{D})$ show rat SC specimens after ex vivo imaging with a $9.4 \mathrm{~T}$ MRI scanner and after treatment in DSI studio software for tractography. $A, S C$ from a rat subjected to dorsal root avulsion and subsequent replantation into the dorsolateral spinal cord. This animal received RAR $\beta$ treatment and tractography using the dorsolateral white matter as the origin for the tracing-indicated connection between the $\mathrm{SC}$ and the replanted dorsal root (white arrows). $\boldsymbol{B}$, In contrast, in an animal treated with the control vehicle, there was no indication of connections between the white matter and the replanted root. $C, D$, Examples of tractography when the entire ipsilateral white matter was used as the origin for tractography in a drug-treated animal $(\boldsymbol{C})$ and a vehicle-treated animal (D). Note the ventral root emerging in $\boldsymbol{C}$. The arrows in $\boldsymbol{C}$ and $\boldsymbol{D}$ indicate the position of the replant site. The color code for fibers running longitudinal to the spinal cord is blue, whereas fibers running perpendicular into the replant appear in green and a sagittal direction is represented in red. 
A

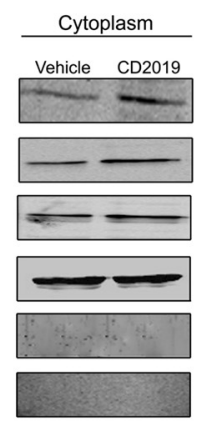

\section{C}
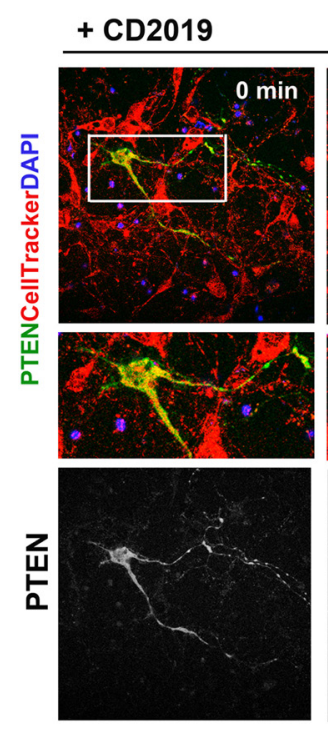
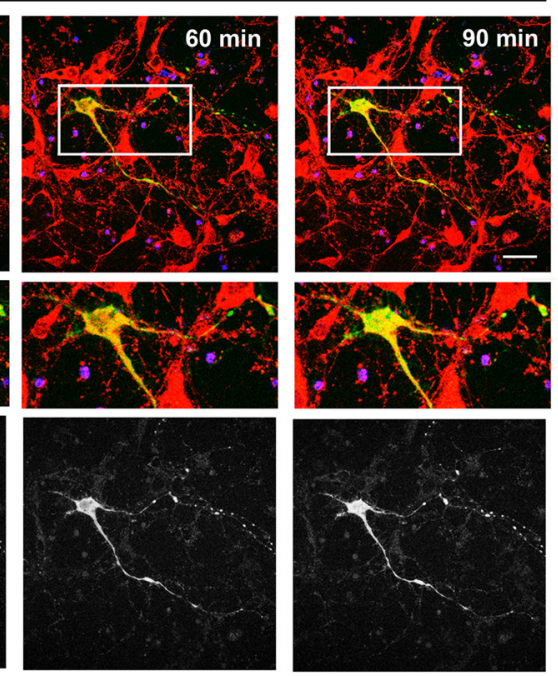

Nucleus

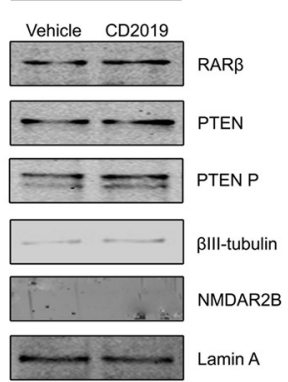

B

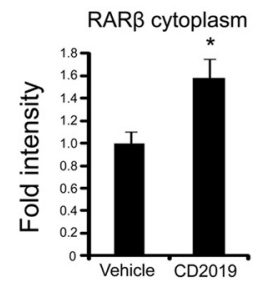

PTEN cytoplasm

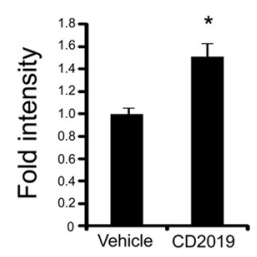

PTEN P cytoplasm

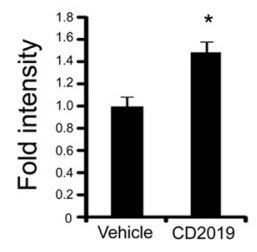

RAR $\beta$ membrane

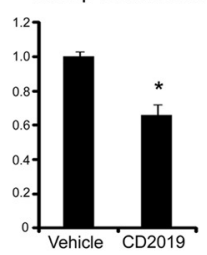

PTEN membrane

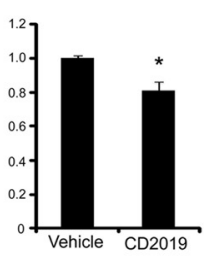

PTEN P membrane

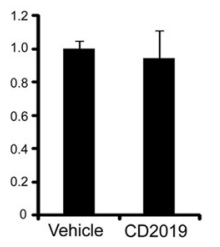

RAR $\beta$ nucleus

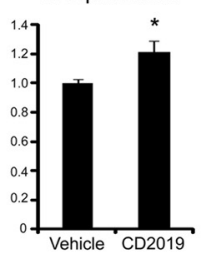

PTEN nucleus

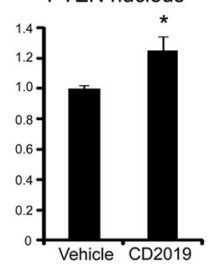

PTEN P nucleus

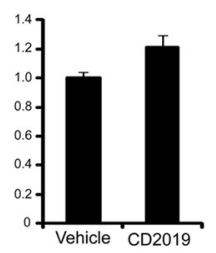

Figure 6. Movement of RAR $\beta$ and PTEN in response to $72 \mathrm{~h}$ treatment with the RAR $\beta$ agonist CD2019. A, Mouse cortical neurons ( 0 DIV) were treated with CD2019 (0.1 $\mu \mathrm{m}$ ) for $72 \mathrm{~h}$ and then processed for cell fractionation and analyzed by Western blotting. Levels of both RAR $\beta$ and PTEN are reduced in the membrane fraction in cells treated with CD2019 versus vehicle control. $\boldsymbol{B}$, Quantitative analysis of protein levels are mean values expressed as fold of vehicle (independent-samples $t$ test, ${ }^{*} p \leq 0.05$ ). $n=4$ for all experiments. Error bars indicate \pm SEM. C, CD2019 induces a rapid (visible at $60 \mathrm{~min}$ ) movement of PTEN into the cytoplasm in cortical neurons. Insets illustrate the shift of PTEN from the neurites into the cell body. Scale bar, $20 \mu \mathrm{m}$.

Stoorvogel, 2013), we wanted to verify that this was a PTENmediated event. To test this, neurons were transfected with a PTEN siRNA or a scrambled siRNA and cultured with either vehicle or CD2019. After $72 \mathrm{~h}$, exosomes were extracted from the medium and Western blotting confirmed that PTEN was knocked in both cell lysates and in the exosomes from cultures treated with the PTEN siRNA (Fig. $8 A, B$ ). This was further corroborated by immunostaining of glial cultures treated with these exosomes for $24 \mathrm{~h}$ (Fig. 8 $C, D)$. Next, we treated astrocytes for $24 \mathrm{~h}$ with PTEN-depleted (from PTEN-siRNA-transfected neurons) or nondepleted (from scrambled-siRNA-transfected neurons) exosomes from CD2019treated cultures and found that only the latter induced a significant reduction in cell proliferation (Fig. 8E). This suggests that PTEN plays a crucial role in modulating astrocyte proliferation.

To determine whether this paradigm was sustained in vivo, a pool of exosomes was injected directly at the site of reimplantation of avulsed dorsal roots into the SC (C5-C8, T1), with a group of animals receiving exosomes from CD2019-treated neurons; the other group received exosomes from vehicle-treated cultures (Fig. 9A). Confirmation of the appropriate target delivery of exosomes was obtained by immunostaining of tissue removed from a subset of animals of both groups that were killed the day after surgery. Remarkably, analysis of serial longitudinal sections taken from $100 \mu \mathrm{m}$ each side of the implantation/exosome injection site showed a dramatic increase of PTEN in astrocytes in animals that were injected with CD2019-treated neuronal exosomes (Fig. 9B). To assess the effect of this boost of PTEN in astrocytes, we looked at differences in the glial population after 2 weeks, when the glial scar would have formed (Cregg et al., 2014). It is noteworthy to point out that we only looked at the astrocytic component of the scar and did not assess other cell types that typically populate the scar core (Cregg et al., 2014). There was a clear difference in GFAP-positive density population and morphology between the two groups. The animals that received exosomes from $\mathrm{RAR} \beta$-agonist-treated neuronal cultures did not have an increased astrocyte population around the injury site on either side of the DREZ and the astrocytes had long, thin, unbranched processes resembling resting local fibrous glia (Fig. 9C). The group treated with exosomes from vehicle-cultured neurons showed a much denser population of astrocytes on either side of the DREZ that were morphological strikingly different in that a mixture of radial and elongated phenotypes were present with hypertrophic processes that were entangled in each other (Fig. 9C). These are known to act as a major physical barrier to regenerating axons (Wanner et al., 2013). However, when we looked at regeneration after 6 weeks of exosome treatment, we did not find any BDAlabeled axons crossing into the SC in animals that received exosomes from RAR $\beta$-agonist-treated neurons, in which, despite phenotypic features of a glial scar at the injury site being absent, no colabeled $\beta$ III tubulin-BDA axons could be seen crossing the DREZ (Fig. 9D). 
A

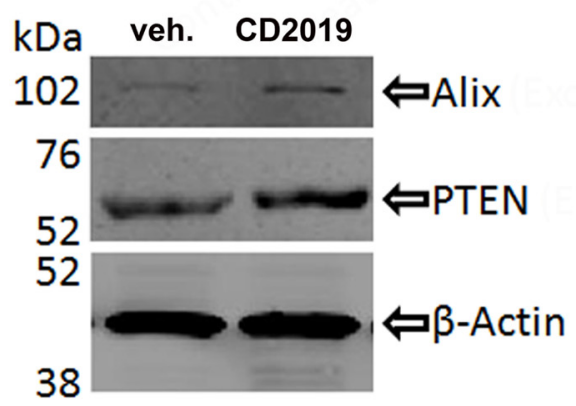

B

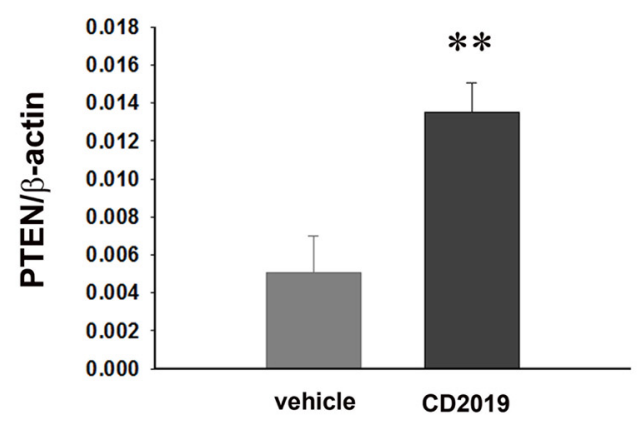

C

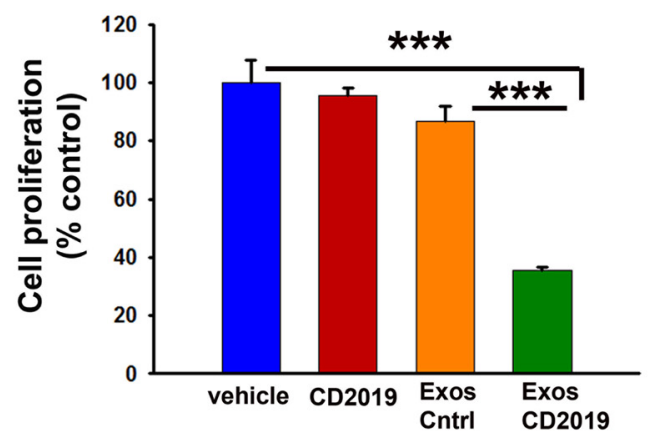

$\mathbf{F}$

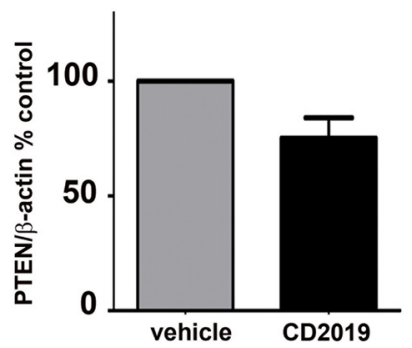

D
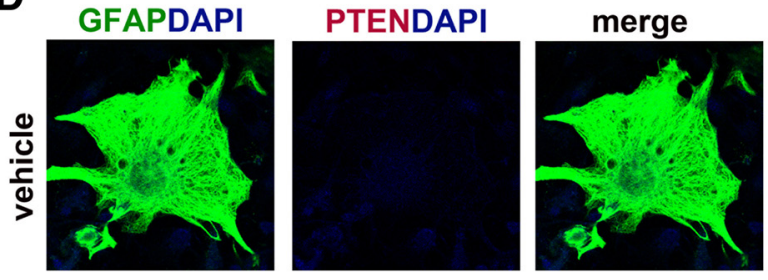

GFAPPTEN
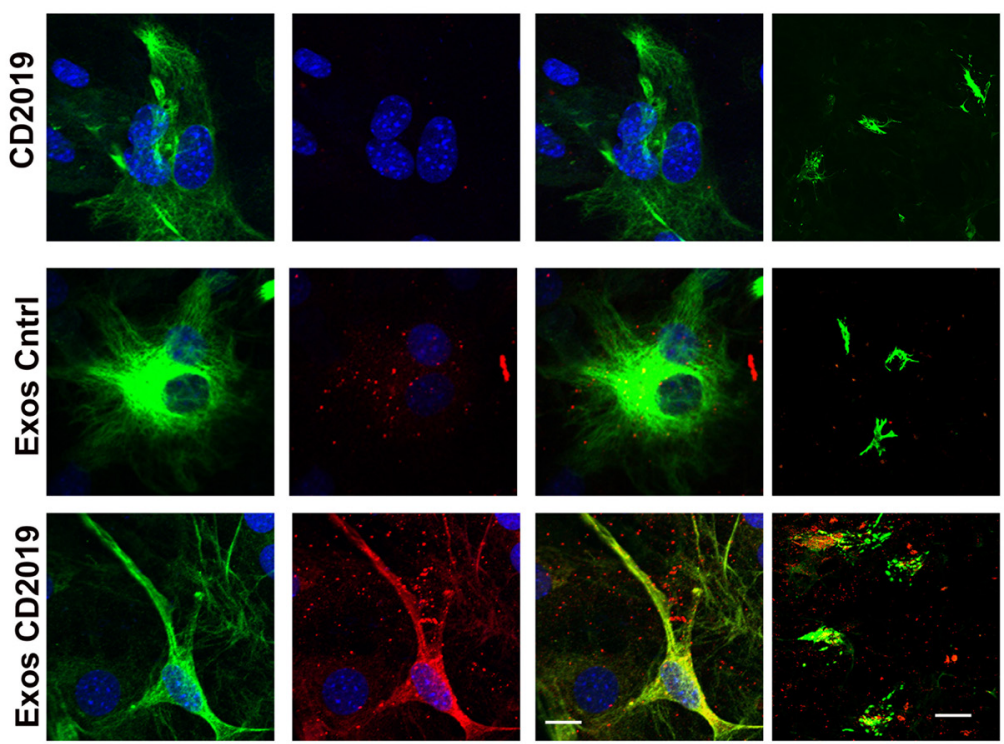

E

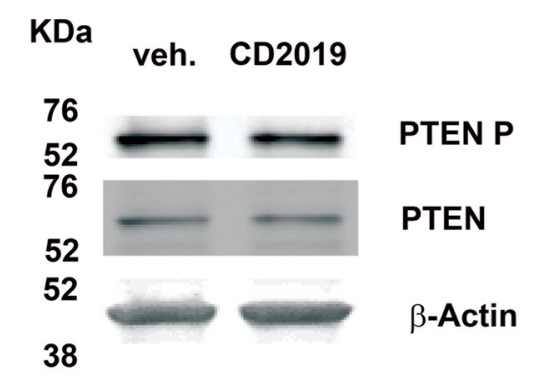

G
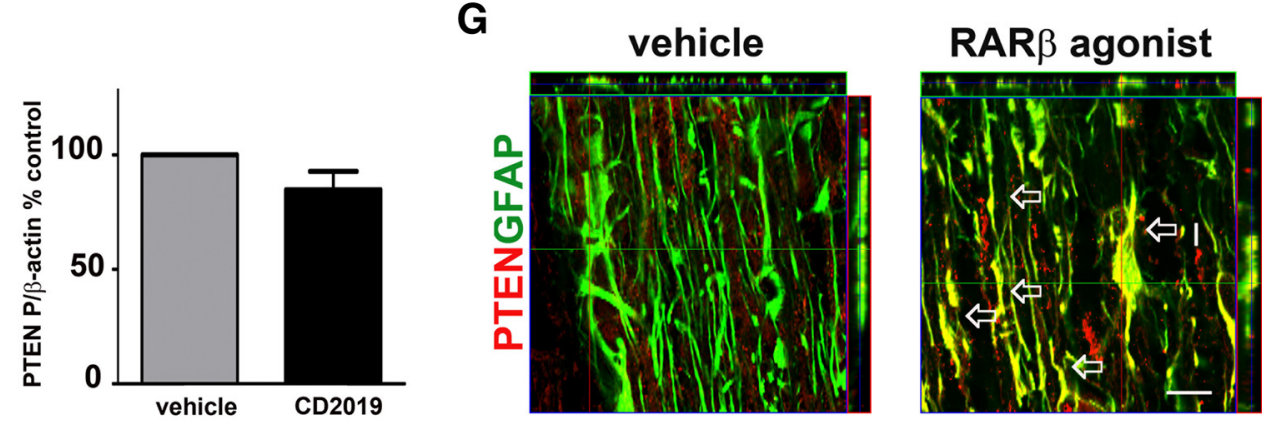

Figure 7. RAR $\beta$ agonist modulates astrocyte proliferation via induction of PTEN secretion by neurons in exosomes. $\boldsymbol{A}, \boldsymbol{B}$, Western blots showing that PTEN is present in exosomes secreted by neurons $(\boldsymbol{A})$ and is enriched in exosomes derived from $\operatorname{CD} 2019(0.1 \mu \mathrm{M})$-treated neurons $(\boldsymbol{B})$. Figure shows a ratio of exosomal PTEN to neuronal $\beta$-actin. ${ }^{* *} p \leq 0.01$, Student's $t$ test. $C$, Cell proliferation assay showing that neuronal exosomes enriched in PTEN hamper astrocyte proliferation in vitro. Data represent mean $\pm S E M .{ }^{* * *} p \leq 0.001$, one-way ANOVA followed by Tukey's test. D, PTEN is visible only in astrocytes treated with exosomes derived from (D2019-treated neurons. Scale bar, 20 and $50 \mu \mathrm{m}$ for the last column. $\boldsymbol{E}, \boldsymbol{F}$, There is no change in PTEN nor phosphorylated PTEN (PTEN P) in astrocytes treated with CD2019 compared with vehicle-treated cultures, Data show mean \pm SEM, Student's $t$ test. G, PTEN is present in astrocytes in the SC of RAR $\beta$-agonist-treated rats but absent in vehicle-treated ones. Scale bar, $20 \mu \mathrm{m}$. 
A

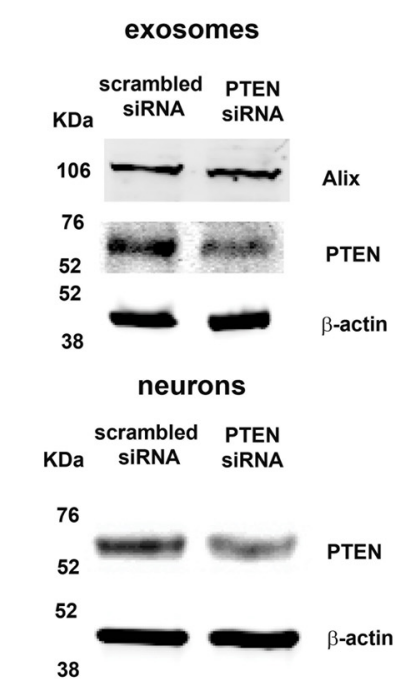

D

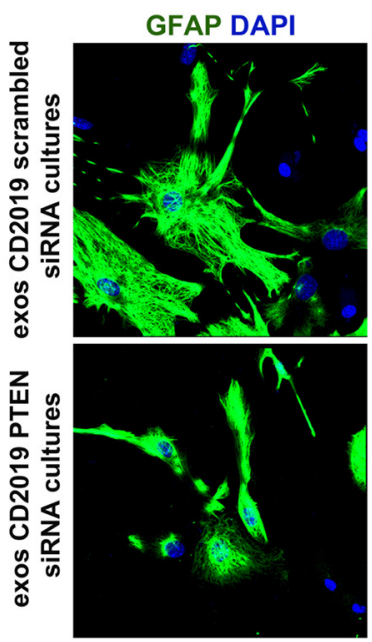

B
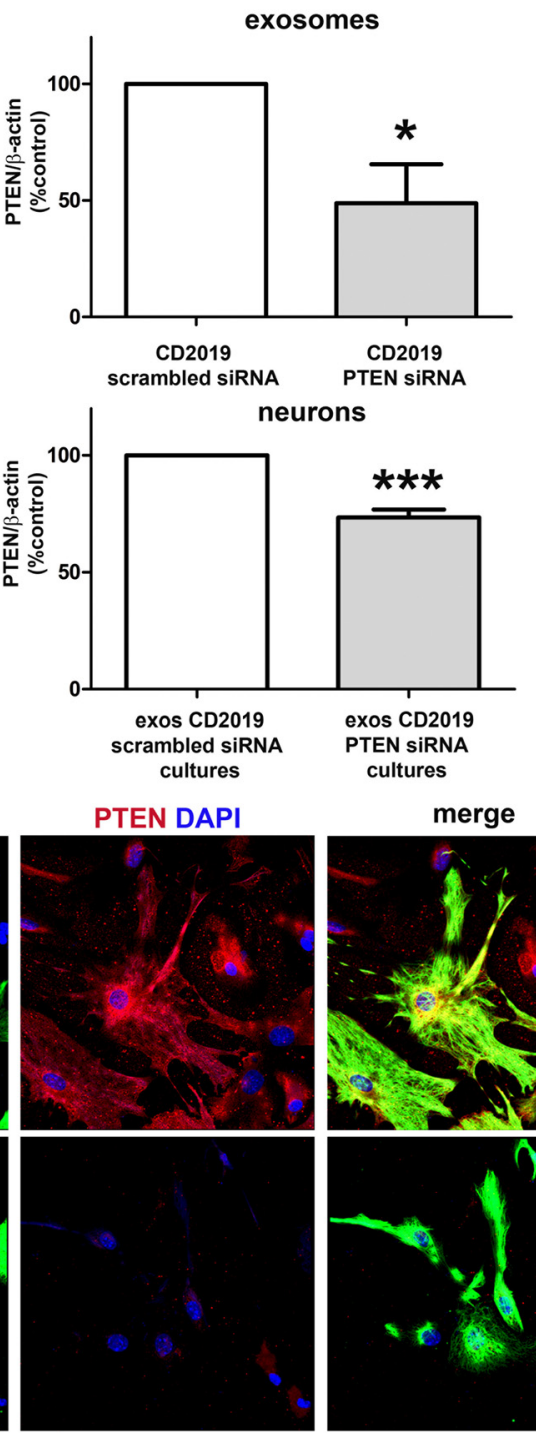

C

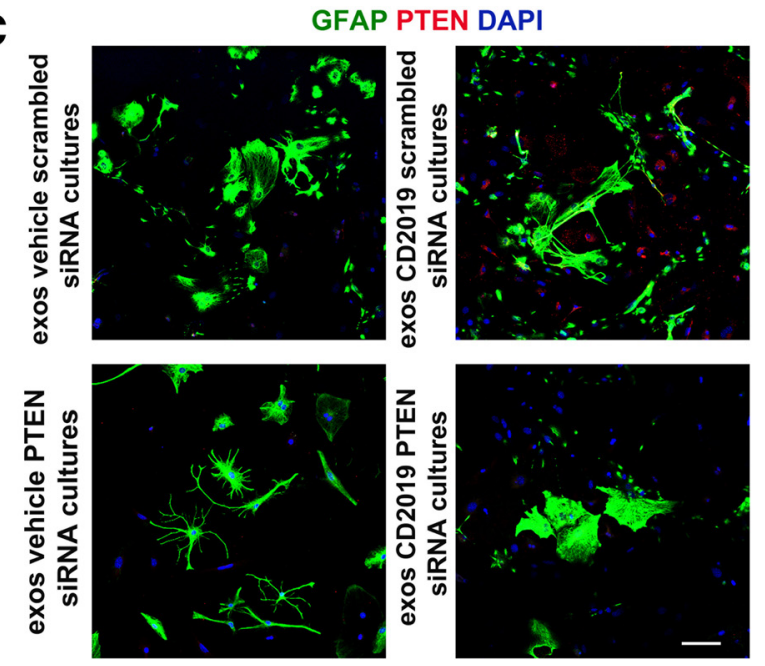

$\mathbf{E}$

Figure 8. Loss of PTEN prevents neuronal exosome mediated modulation of astrocyte proliferation. $\boldsymbol{A}$, Western blots showing that transfection of cortical neurons with PTEN siRNA resulted in a loss of PTEN in the secreted exosomes (top) and in the cell lysates (bottom). $\boldsymbol{B}$, Quantitative analysis of protein levels are mean values expressed as fold of control (scrambled siRNA) after normalization for neuronal $\beta$-actin. ${ }^{*} p \leq 0.05 ;{ }^{* * *} p \leq 0.001$, Student's $t$ test. $n=3$ for all experiments. Error bars indicate \pm SEM. $C$, Expression of PTEN in astrocytes cultured for $24 \mathrm{~h}$ with exosomes from scrambled or PTEN-siRNA-transfected neurons in the presence or absence of CD2019. Scale bar, $100 \mu \mathrm{m}$. D, High-magnification images showing that high levels of PTEN could only be seen in cultures treated with exosomes from scrambled-siRNA-transfected neurons treated with CD2019. Scale bar, $50 \mu \mathrm{m}$. $E$, Cell proliferation assay showing that only exosomes enriched in PTEN (from CD2019-scrambled siRNA-transfected neurons) modulate astrocyte proliferation in. Data represent mean \pm SEM. ${ }^{* * *} p \leq$ 0.001 , one-way ANOVA followed by Tukey's test.

Because neurons also secrete exosomes and these can represent a mechanism of interneuronal communication (Chivet et al., 2014), we next assessed whether the lack of axonal growth could be due to interneuronal exosome exchange. Neurite outgrowth was measured in cortical neurons treated with vehicle or CD2019 or exosomes from neuronal cultures that had been treated with either vehicle or CD2019 for $72 \mathrm{~h}$. We found that only CD2019 caused a significant increase in neurite outgrowth, with all of the other treatments having no effect compared with control cultures (Fig. 9E). This not only rules out a negative effect of interneuronal exosome exchange on neuronal growth programs, but also emphasizes that it is the RAR $\beta$ agonist modulation that is crucial.

Next, we sought to confirm that the increase in astrocytic PTEN observed in the avulsed rats that received PTEN-enriched exosome injections resulted from the uptake of those exosomes rather than from an increase in endogenous expression. Therefore, we treated avulsed rats with an exosome inhibitor alone (GW4869, $1 \mathrm{mg} / \mathrm{kg}$ ) and in conjunction with RAR $\beta$ agonist for 4 weeks. We then looked at PTEN expression 2 and 4 weeks later and compared it with the one seen in $\operatorname{RAR} \beta$-agonist-treated rats for the same period of time. We confirmed that the origin of the PTEN in astrocytes of rats treated with the RAR $\beta$ agonist derives essentially from the exosomes because there was little or no PTEN in astrocytes in either GW4869-treated group at 2 weeks (Fig. $10 A$ ) and 4 weeks (Fig. 10B), but PTEN was abundant in astrocytes of RAR $\beta$-agonist-treated rats after 2 weeks (Fig. 10A) and 4 weeks of treatment (Fig. 7E). Concurrently, the effects in glial cells elicited by the exosome-derived PTEN were also absent upon treatment with the exosome inhibitor and a glial scar with densely entangled astrocytes at the DREZ was seen at 2 and 4 


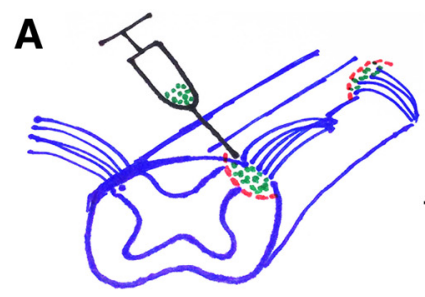

B
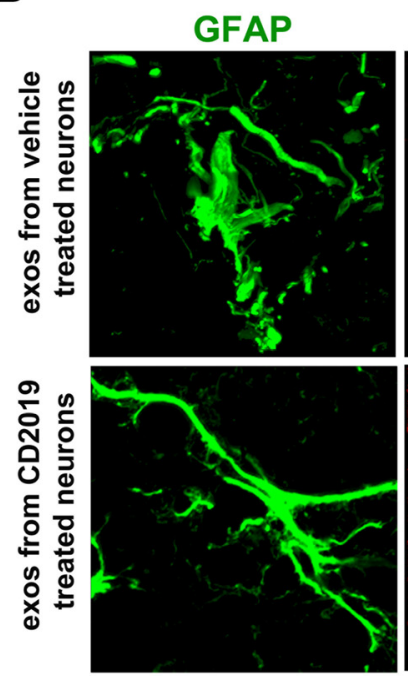

C
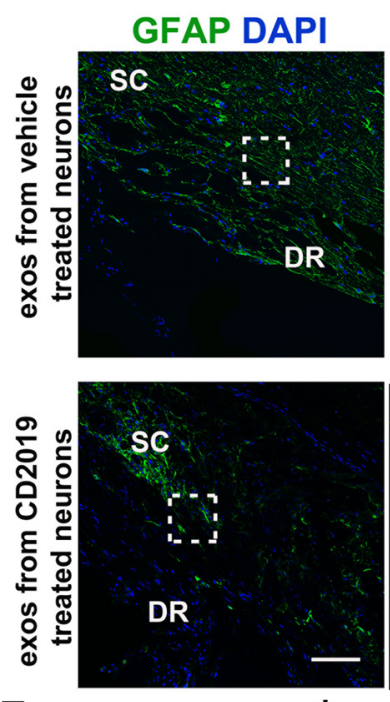

E

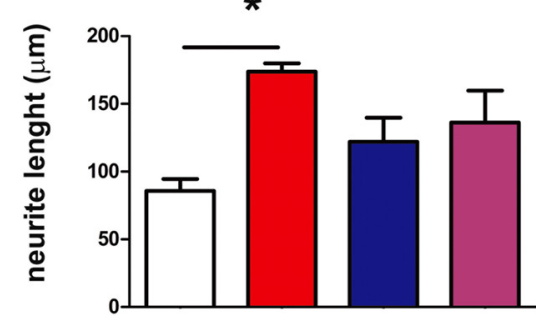

PTEN in

astrocytes

at exosome

1 day

injection site 2 weeks scar 6 weeks
BDA labelled

axons in

spinal cord

\section{1 day after surgery}
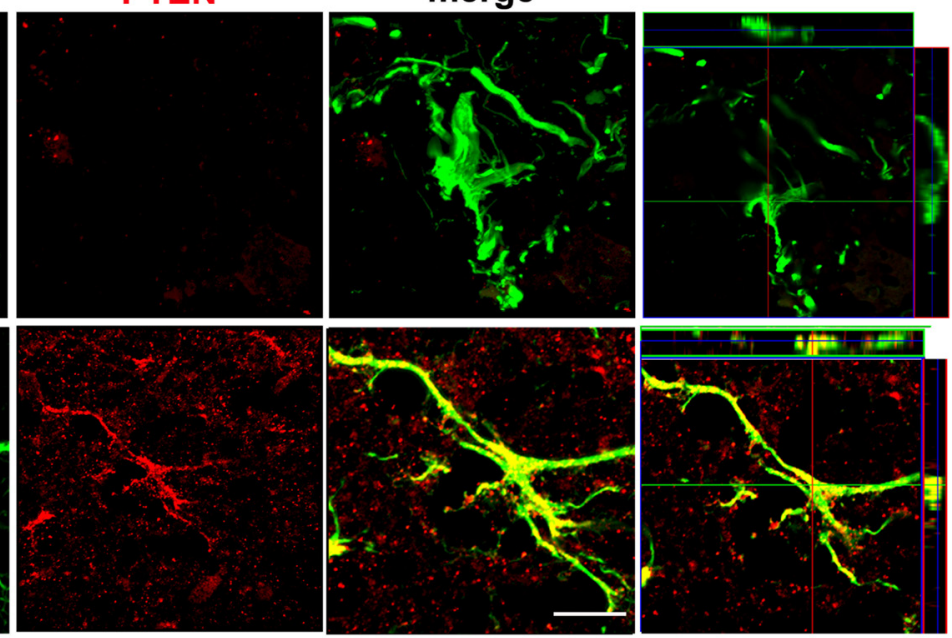

D

6 weeks after surgery
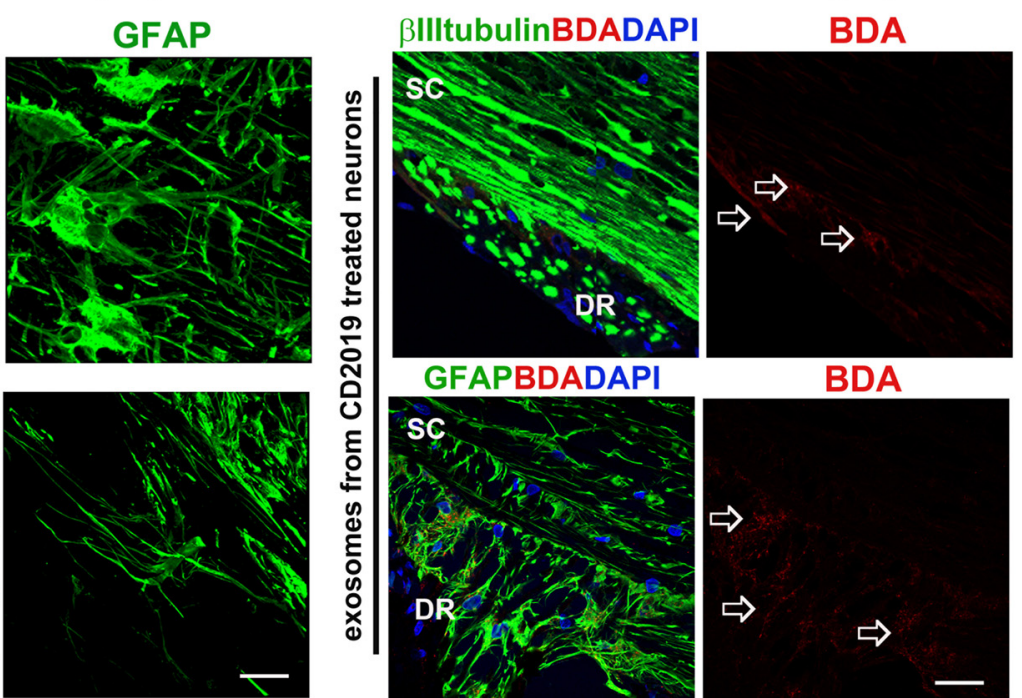

GFAPBDADAPI
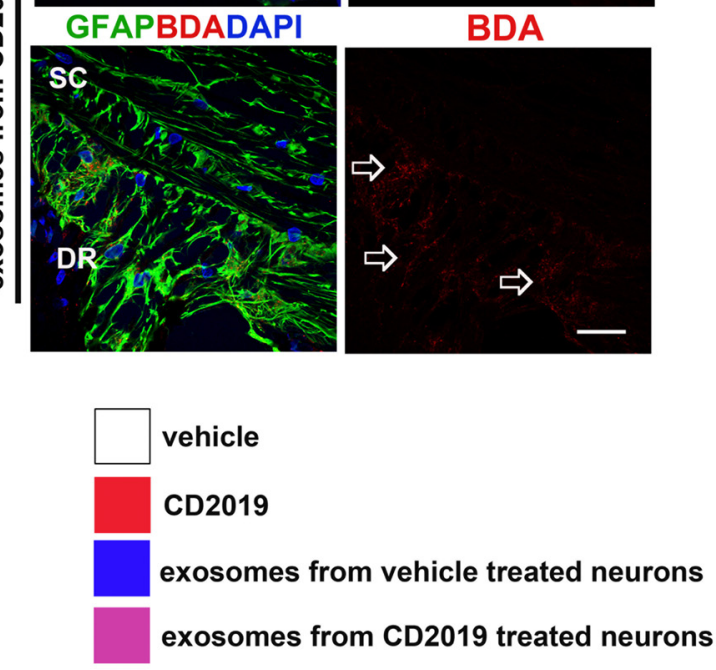

Figure 9. PTEN-enriched neuronal exosomes prevent scar formation after avulsion. $A$, Illustration of experimental design: exosomes isolated from CD2019- or vehicle-treated neurons were injected into the pocket where the severed dorsal roots were reimplanted at the time of surgery. $\boldsymbol{B}$, One day later, the presence of PTEN in astrocytes was assessed at the injection site and found to have been taken up by astrocytes in animals that received PTEN-enriched exosomes. Scale bar, $15 \mu \mathrm{m}$. C, Differences in astrocyte density and morphology at the reimplantation site between the two groups. Right images (scale bar, $20 \mu \mathrm{m}$ ) are insets from the images on the left (scale bar, $100 \mu \mathrm{m}) \cdot \boldsymbol{D}$, BDA-labeled axons at 6 weeks after surgery do not enter the SC and come to a stop before the DREZ (arrows). Scale bar, $100 \mu \mathrm{m}$. $\boldsymbol{E}$, Treatment of cortical neurons with neuronal exosomes from cultures that had been treated either with vehicle or CD2019 had no effect in neurite outgrowth. Only treatment with the RAR $\beta$ agonist caused a significant increase in neurite length. Data represent mean \pm SEM. ${ }^{*} p<0.05$, one-way ANOVA followed by Tukey's test. 
A
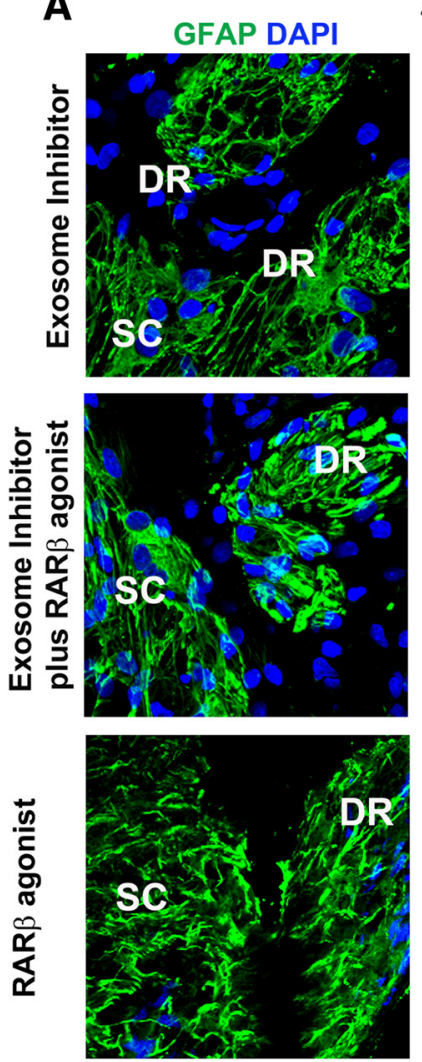

B
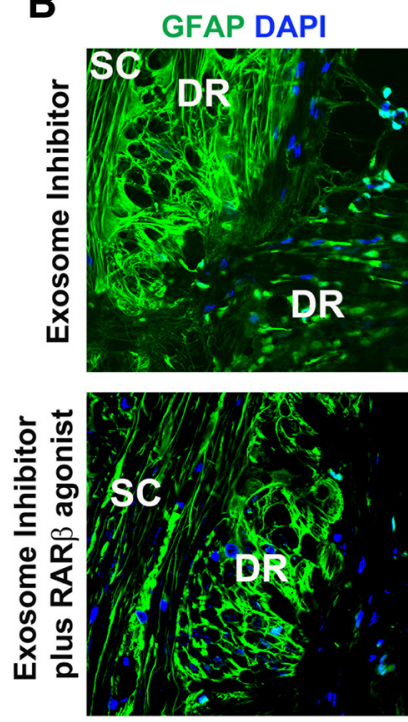

C

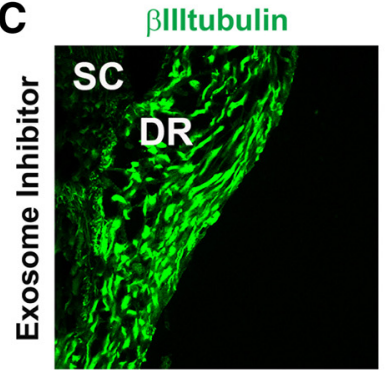

\section{2 weeks post-surgey}
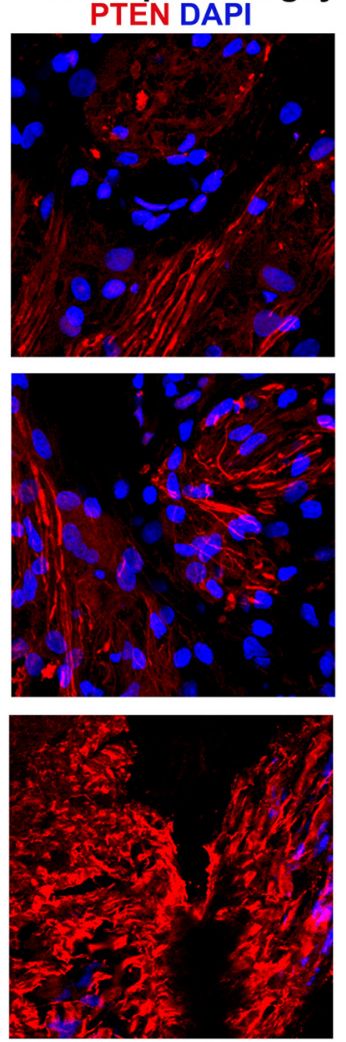

4 weeks post-surgey PTEN DAPI
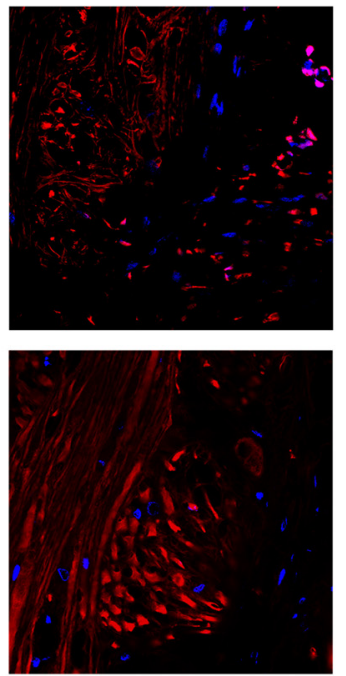

PTEN

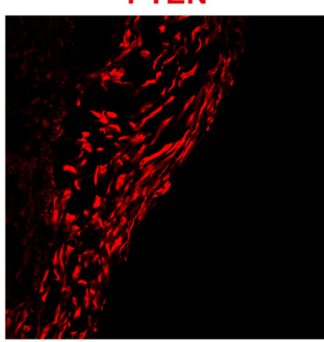

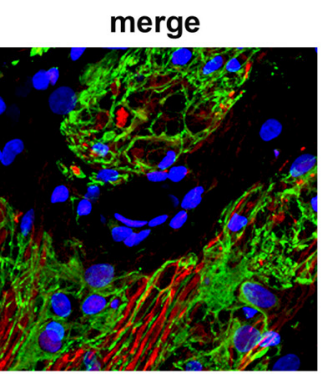
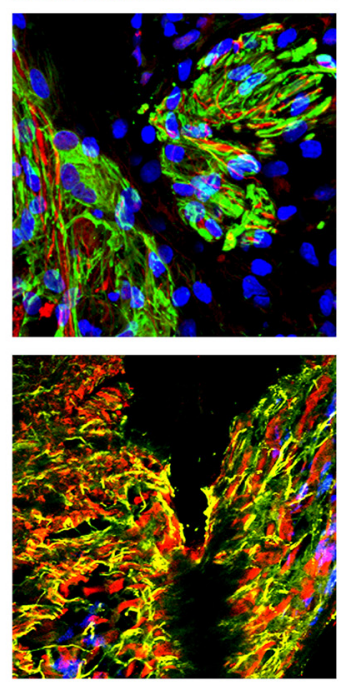

merge
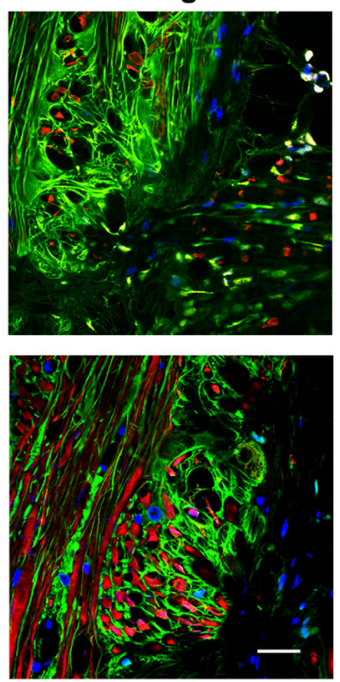

merge

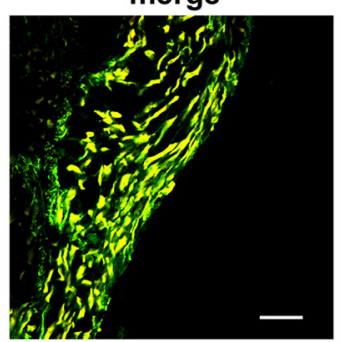

Figure 10. Exosome inhibition prevents RAR $\beta-P T E N$ - induced modulation of the glial scar after avulsion. Avulsed rats were treated with GW4869 alone or GW4869 and RAR $\beta$ agonist for 4 weeks after surgery. $A$, After 2 weeks of exosome inhibitor treatment, PTEN could not be seen in astrocytes surrounding the lesioned area and a glial scar is present. In contrast, RAR $\beta$ agonist-treated rats show a considerable amount of PTEN in astrocytes and these do not form an entangled mesh, but rather

weeks after surgery (Fig. 10A,B). Predictably, inhibition of PTEN release in exosomes resulted in PTEN being mainly located in the axons (Fig. 10C).

\section{Discussion}

True axonal regeneration after SC injury has been difficult to verify due to the nature of the models used, which convey limited clinical translational applicability because the lesion induced in the animal is not equivalent to the human injury. In this study, we have modeled in the rat a human root avulsion that is usually inflicted by severe trauma to the forequarter in road traffic accidents affecting mainly young individuals. Here, we describe a suitable animal model and a signaling pathway that reprograms adult neuronal paths and modulates glial activity, allowing regeneration to occur. We identify a novel mechanism of action for PTEN in which it exerts a biological activity in astrocytes via neuronal exosome transfer to prevent scar formation.

A dorsal root injury, although by definition situated in the PNS, is in effect an SC or CNS lesion. Most previous studies aimed at resolving the common human traumatic clinical condition of root avulsion from the SC have investigated the effect of crushing the dorsal root, which is not relevant as an experimental paradigm (Wong et al., 2006). In this type of lesion, it cannot be ruled out that spared axons that sprout and lead to functional output are present. The fact that a bridge to the cord is maintained may allow a mechanism of axonal regeneration to occur unrelated to the human injury, which is a severing of the sensory axons entering the SC. To overcome these limitations, we modeled in the rat a human avulsion injury. The roots were not exactly avulsed or pulled out from their attachment with the SC, but rather cut at the SC surface. There is a certain difference between the two traumas in that, with a true avulsion, there would be some more secondary changes within the SC, such as bleeding and cell death (Chew et al., 2011). However, the avulsion trauma is much more difficult to standardize with regard to magnitude of power of pull, direction, and so forth than a clean cut, so the effect on the integrity of

$\leftarrow$

distribute in an elongated manner toward the SC. $\boldsymbol{B}$, The same pattern of expression of PTEN in astrocytes seen at 2 weeks is observed at 4 weeks after surgery for both groups treated with GW4869. Scale bar, $100 \mu \mathrm{m}$. C, Treatment with exosome inhibitor for 4 weeks induces PTEN to remain in the axons. Scale bar, $100 \mu \mathrm{m}$. 
dorsal root axons would be the same. After dorsal root cut and subsequent implantation into the SC, without any adjuvant therapy, there is only minimal or abortive growth of dorsal root axons in the SC (Carlstedt, 1985; Siegal et al., 1990). In our avulsion system, we were able to show that activation of $\operatorname{RAR} \beta$ signaling induces regeneration of severed axons.

Of paramount importance is how the regenerated dorsal root axons re-enter the SC. In the naive adult situation, there is a specialized "transitional region" between a stereotype peripheral nerve compartment of the dorsal root and the central nervous fiber tracts of the SC, which involves a number of unique structural entities (Berthold and Carlstedt, 1977a). Among these are the occurrence of fibrous astrocytic processes surrounding and separating the most proximal peripheral nervous parts of the myelinated fibers, as well as the formation of the compound PNS-CNS type of node of Ranvier at the crossing of nerve fibers from the PNS to the CNS type of organization (Berthold and Carlstedt, 1977b). Here, we show that the regrowing dorsal root nerve fibers extend along a pathway very much resembling the normal dorsal root PNS-CNS transitional region where fibrous astrocytic processes encase the most proximal PNS paranode of individual myelinated fibers. It is also obvious from the present results that there are compound PNS-CNS nodes of Ranvier as the myelinated nerve fibers pass from the dorsal root into the SC. These results suggest that reconstituting a naive tissue organization at the PNS-CNS interface is important to allow for nerve fiber regeneration into the SC and the CNS, but this new observation has to be tested and studied further.

Dorsal root nerve fiber regeneration was also shown by BDAlabeled axons crossing through the DREZ, as well as various proteins that are upregulated during regeneration and involved in synaptic activity across the SC and/or dorsal root. In addition, images of tractography supported the possibility of a robust growth of axons from the replanted dorsal root into the superficial dorsolateral white matter on the lesioned side of the SC. Hypothetically, the same type of signal could be due to movements of water in glial processes. However, it seems less likely that individual glial processes actually would be organized in parallel bundles extending from the PNS compartment of the dorsal root into the dorsal funiculus. Collectively, these data suggest that axonal regeneration took place and new connections were made in the dorsal horn. This indicates a switch from the nonpermissive environment that sets after SCI into a favorable one. Here, we show that RAR $\beta$ agonism modulates the PTEN signaling pathway in neurons and in astrocytes. In neurons, $\operatorname{RAR} \beta$, via a cytoplasmic effect, induces PTEN to move from the membrane, where it blocks axonal outgrowth via PI3K inhibition (Park et al., 2010), into the cytoplasm, where it becomes phosphorylated and hence inactive (Ross and Gericke, 2009). In addition, stimulation of RAR $\beta$ results in an increased secretion of PTEN in exosomes. Recruitment of PTEN into exosomes requires Ndfip 1 and PTEN ubiquitination (Putz et al., 2012), which are also required for nuclear translocation of PTEN (Trotman et al., 2007; Howitt et al., 2012). Although we did not look directly at these two factors, we saw a significant increase of nuclear PTEN in response to RAR $\beta$ agonist treatment. Given that PTEN recruitment into both exosomes and the nucleus are governed by the same biological factors, it stands to reason that RAR $\beta$ might induce the release of PTEN-enriched exosomes, at least in part via ubiquitination and enrollment of Ndfip 1. In agreement with this, it has been shown that RA induces PTEN ubiquitination (Song et al., 2008), placing this mechanism as a likely candidate for the generation of neuronal PTEN-enriched exosomes in response to $\mathrm{RAR} \beta$ agonist treatment. In astrocytes, $\mathrm{RAR} \beta$ signaling does not modulate PTEN in a direct manner, having no effect on endogenous or exosome-secreted PTEN. Instead, it increases PTEN activity in astrocytes via external uptake from neuronal exosomes. Only recently has the importance of exosomes in fine-tuned cell-to-cell communication come to light (Chivet et al., 2013). We have identified a mechanism that elucidates the cross talk between neurons and astrocytes, in which PTEN is secreted from neurons in exosomes that are taken up by astrocytes. This appears to be a physiological event because PTEN was found in exosomes from vehicle-treated neurons, but CD2019-treated cultures released exosomes significantly enriched in PTEN. A similar non-cell-autonomous mode of PTEN activity has been described recently in which PTEN secreted in exosomes alters the physiological state of the recipient cells (Putz et al., 2012). This sheds new light into the PTEN function in adult regeneration, which has been largely perceived as solely inhibitory (Park et al., 2010).

This exosome-derived boost in PTEN that reaches the astrocytes is enough to hamper their proliferation, thus preventing the formation of a glial scar. PTEN negatively regulates PI3K/AKt signaling and various studies in non-neuronal cells have identified mTOR as a key downstream signal of PI3K/AKt in regulating cellular growth (Sabatini, 2006; Abramov et al., 2009). The Rheb-mTOR pathway is upregulated in reactive astrocytes of the injured SC (Codeluppi et al., 2009), as is p-PTEN (inactive form) and p-AKt (active) in reactive astrocytes in the rat ischemic hippocampus (Choi et al., 2005). It is therefore likely that this is the pathway by which increased PTEN in astrocytes reduces gliosis and scar size. Accordingly, we observed that treatment with RAR $\beta$ agonist or PTEN-enriched exosomes (injected at the injured site) resulted in a remarkably reduced scar and a normal astrocytic phenotype. When the agonist was administered to rats with an exosome inhibitor and when exosomes extracted from neurons cultured in the presence of a PTEN siRNA were added to glial cultures, the antiproliferative/glial modulation effects disappeared, corroborating our proposed signaling pathway of RAR $\beta$ induced neuron-glial PTEN transfer in exosomes. Interestingly, when rats received PTEN-enriched exosomes at the injury site (and no agonist treatment), there was no improvement in axonal regeneration. We did not investigate here whether these exosomes also target and modulate other cell types involved in the formation of the scar core, such as NG2 cells, microglia, and blood-borne macrophages (Cregg et al., 2014), but the considerable reduction/absence of a glial scar certainly suggests this as a plausible situation. The lack of axonal regeneration despite the absence of a glial scar may be attributed to either the limited dose of PTEN-enriched exosomes (just one injection); alternatively, to prevent scar formation, it may simply not be not enough to induce sustained axonal regeneration in the adult and a concomitant reprogramming of axonal internal mechanisms may also be required.

In essence, we show here how neuronal RAR $\beta$ signaling modulates both neuronal and glial activity via the PTEN signaling pathway, resulting in a permissive milieu that allows axonal regeneration and functional recovery after cervical avulsion.

\section{References}

Abramov E, Dolev I, Fogel H, Ciccotosto GD, Ruff E, Slutsky I (2009) Amyloid-beta as a positive endogenous regulator of release probability at hippocampal synapses. Nat Neurosci 12:1567-1576. CrossRef Medline

Agudo M, Yip P, Davies M, Bradbury E, Doherty P, McMahon S, Maden M, Corcoran JP (2010) A retinoic acid receptor beta agonist (CD2019) overcomes inhibition of axonal outgrowth via phosphoinositide3-kinase signalling in the injured adult spinal cord. Neurobiol Dis 37:147-155. CrossRef Medline

Alvarez FJ, Titus-Mitchell HE, Bullinger KL, Kraszpulski M, Nardelli P, Cope TC (2011) Permanent central synaptic disconnection of proprioceptors after nerve injury and regeneration. I. Loss of VGLUT1/IA synapses on motoneurons. J Neurophysiol 106:2450-2470. CrossRef Medline

Berthold CH, Carlstedt T (1977a) Observations on the morphology at the transition between the peripheral and the central nervous system in the 
cat. II. General organization of the transitional region in S1 dorsal rootlets. Acta Physiol Scand Suppl 446:23-42. Medline

Berthold CH, Carlstedt T (1977b) Observations on the morphology at the transition between the peripheral and the central nervous system in the cat. III. Myelinated fibres in S1 dorsal rootlets. Acta Physiol Scand Suppl 446:43-60. Medline

Berthold CH, Carlstedt T, Corneliuson O (1993) The central-peripheral transition zone, Vol 1, Ed 3. In: Peripheral neurophathy (Dyck PJ, Thomas PK, eds), pp 73-91. Philadelphia: WB Saunders.

Bradbury EJ, Moon LD, Popat RJ, King VR, Bennett GS, Patel PN, Fawcett JW, McMahon SB (2002) Chondroitinase ABC promotes functional recovery after spinal cord injury. Nature 416:636-640. CrossRef Medline

Bush TG, Puvanachandra N, Horner CH, Polito A, Ostenfeld T, Svendsen CN, Mucke L, Johnson MH, Sofroniew MV (1999) Leukocyte infiltration, neuronal degeneration, and neurite outgrowth after ablation of scarforming, reactive astrocytes in adult transgenic mice. Neuron 23:297-308. CrossRef Medline

Carlstedt T (1985) Dorsal root innervation of spinal cord neurons after dorsal root implantation into the spinal cord of adult rats. Neurosci Lett 55:343-348. CrossRef Medline

Carracedo A, Pandolfi PP (2008) The PTEN-PI3K pathway: of feedbacks and cross-talks. Oncogene 27:5527-5541. CrossRef Medline

Chew DJ, Carlstedt T, Shortland PJ (2011) A comparative histological analysis of two models of nerve root avulsion injury in the adult rat. Neuropathol Appl Neurobiol 37:613-632. CrossRef Medline

Chivet M, Javalet C, Hemming F, Pernet-Gallay K, Laulagnier K, Fraboulet S, Sadoul R (2013) Exosomes as a novel way of interneuronal communication. Biochem Soc Trans 41:241-244. CrossRef Medline

Chivet M, Javalet C, Laulagnier K, Blot B, Hemming FJ, Sadoul R (2014) Exosomes secreted by cortical neurons upon glutamatergic synapse activation specifically interact with neurons. J Extracell Vesicles 3:24722. Medline

Choi JS, Park HJ, Kim HY, Kim SY, Lee JE, Choi YS, Chun MH, Chung JW, Lee MY (2005) Phosphorylation of PTEN and Akt in astrocytes of the rat hippocampus following transient forebrain ischemia. Cell Tissue Res 319: 359-366. CrossRef Medline

Codeluppi S, Svensson CI, Hefferan MP, Valencia F, Silldorff MD, Oshiro M, Marsala M, Pasquale EB (2009) The Rheb-mTOR pathway is upregulated in reactive astrocytes of the injured spinal cord. J Neurosci 29: 1093-1104. CrossRef Medline

Corcoran J, So PL, Barber RD, Vincent KJ, Mazarakis ND, Mitrophanous KA, Kingsman SM, Maden M (2002) Retinoic acid receptor beta2 and neurite outgrowth in the adult mouse spinal cord in vitro. J Cell Sci 115: 3779-3786. CrossRef Medline

Corcoran J, Shroot B, Pizzey J, Maden M (2000) The role of retinoic acid receptors in neurite outgrowth from different populations of embryonic mouse dorsal root ganglia. J Cell Sci 113:2567-2574. Medline

Cregg JM, DePaul MA, Filous AR, Lang BT, Tran A, Silver J (2014) Functional regeneration beyond the glial scar. Exp Neurol 253:197-207. CrossRef Medline

Cullheim S, Carlstedt T, Lindå H, Risling M, Ulfhake B (1989) Motoneurons reinnervate skeletal muscle after ventral root implantation into the spinal cord of the cat. Neuroscience 29:725-733. CrossRef Medline

Dinkins MB, Dasgupta S, Wang G, Zhu G, Bieberich E (2014) Exosome reduction in vivo is associated with lower amyloid plaque load in the 5XFAD mouse model of Alzheimer's disease. Neurobiol Aging 35: 1792-1800. CrossRef Medline

Faulkner JR, Herrmann JE, Woo MJ, Tansey KE, Doan NB, Sofroniew MV (2004) Reactive astrocytes protect tissue and preserve function after spinal cord injury. J Neurosci 24:2143-2155. CrossRef Medline

Fraser MM, Zhu X, Kwon CH, Uhlmann EJ, Gutmann DH, Baker SJ (2004) Pten loss causes hypertrophy and increased proliferation of astrocytes in vivo. Cancer Res 64:7773-7779. CrossRef Medline

Goncalves MB, Boyle J, Webber DJ, Hall S, Minger SL, Corcoran JP (2005) Timing of the retinoid-signalling pathway determines the expression of neuronal markers in neural progenitor cells. Dev Biol 278:60-70. CrossRef Medline

Goncalves MB, Clarke E, Hobbs C, Malmqvist T, Deacon R, Jack J, Corcoran JP (2013) Amyloid beta inhibits retinoic acid synthesis exacerbating Alzheimer disease pathology which can be attenuated by an retinoic acid receptor alpha agonist. Eur J Neurosci 37:1182-1192. CrossRef Medline

Herrmann JE, Shah RR, Chan AF, Zheng B (2010) EphA4 deficient mice maintain astroglial-fibrotic scar formation after spinal cord injury. Exp Neurol 223:582-598. CrossRef Medline

Howitt J, Lackovic J, Low LH, Naguib A, Macintyre A, Goh CP, Callaway JK, Hammond V, Thomas T, Dixon M, Putz U, Silke J, Bartlett P, Yang B, Kumar S, Trotman LC, Tan SS (2012) Ndfip1 regulates nuclear Pten import in vivo to promote neuronal survival following cerebral ischemia. J Cell Biol 196:29-36. CrossRef Medline

Hunt SP, Pini A, Evan G (1987) Induction of c-fos-like protein in spinal cord neurons following sensory stimulation. Nature 328:632-634. CrossRef Medline

Li XQ, Verge VM, Johnston JM, Zochodne DW (2004) CGRP peptide and regenerating sensory axons. J Neuropathol Exp Neurol 63:1092-1103. Medline

Lopez-Verrilli MA, Picou F, Court FA (2013) Schwann cell-derived exosomes enhance axonal regeneration in the peripheral nervous system. Glia 61:1795-1806. Medline

Maden M (2001) Role and distribution of retinoic acid during CNS development. Int Rev Cytol 209:1-77. CrossRef Medline

Maehama T, Dixon JE (1998) The tumor suppressor, PTEN/MMAC1, dephosphorylates the lipid second messenger, phosphatidylinositol 3,4,5trisphosphate. J Biol Chem 273:13375-13378. CrossRef Medline

Park KK, Liu K, Hu Y, Kanter JL, He Z (2010) PTEN/mTOR and axon regeneration. Exp Neurol 223:45-50. CrossRef Medline

Putz U, Howitt J, Doan A, Goh CP, Low LH, Silke J, Tan SS (2012) The tumor suppressor PTEN is exported in exosomes and has phosphatase activity in recipient cells. Sci Signal 5:ra70. Medline

Raposo G, Stoorvogel W (2013) Extracellular vesicles: exosomes, microvesicles, and friends. J Cell Biol 200:373-383. CrossRef Medline

Ross AH, Gericke A (2009) Phosphorylation keeps PTEN phosphatase closed for business. Proc Natl Acad Sci U S A 106:1297-1298. CrossRef Medline

Sabatini DM (2006) mTOR and cancer: insights into a complex relationship. Nat Rev Cancer 6:729-734. CrossRef Medline

Sanes JR, Yamagata M (1999) Formation of lamina-specific synaptic connections. Curr Opin Neurobiol 9:79-87. CrossRef Medline

Shearer MC, Fawcett JW (2001) The astrocyte/meningeal cell interface-a barrier to successful nerve regeneration? Cell Tissue Res 305:267-273. CrossRef Medline

Siegal JD, Kliot M, Smith GM, Silver J (1990) A comparison of the regeneration potential of dorsal root fibers into gray or white matter of the adult rat spinal cord. Exp Neurol 109:90-97. CrossRef Medline

Silver J, Miller JH (2004) Regeneration beyond the glial scar. Nat Rev Neurosci 5:146-156. CrossRef Medline

Song MS, Salmena L, Carracedo A, Egia A, Lo-Coco F, Teruya-Feldstein J, Pandolfi PP (2008) The deubiquitinylation and localization of PTEN are regulated by a HAUSP-PML network. Nature 455:813-817. CrossRef Medline

Toy D, Namgung U (2013) Role of glial cells in axonal regeneration. Exp Neurobiol 22:68-76. CrossRef Medline

Trotman LC, Wang X, Alimonti A, Chen Z, Teruya-Feldstein J, Yang H, Pavletich NP, Carver BS, Cordon-Cardo C, Erdjument-Bromage H, Tempst P, Chi SG, Kim HJ, Misteli T, Jiang X, Pandolfi PP (2007) Ubiquitination regulates PTEN nuclear import and tumor suppression. Cell 128:141-156. CrossRef Medline

Wanner IB, Anderson MA, Song B, Levine J, Fernandez A, Gray-Thompson Z, Ao Y, Sofroniew MV (2013) Glial scar borders are formed by newly proliferated, elongated astrocytes that interact to corral inflammatory and fibrotic cells via STAT3-dependent mechanisms after spinal cord injury. J Neurosci 33:12870-12886. CrossRef Medline

Williams RR, Henao M, Pearse DD, Bunge MB (2015) Permissive Schwann cell graft/spinal cord interfaces for axon regeneration. Cell Transplant 24:115-131. CrossRef Medline

Wong LF, Yip PK, Battaglia A, Grist J, Corcoran J, Maden M, Azzouz M, Kingsman SM, Kingsman AJ, Mazarakis ND, McMahon SB (2006) Retinoic acid receptor beta 2 promotes functional regeneration of sensory axons in the spinal cord. Nat Neurosci 9:243-250. CrossRef Medline

Yip PK, Wong LF, Pattinson D, Battaglia A, Grist J, Bradbury EJ, Maden M, McMahon SB, Mazarakis ND (2006) Lentiviral vector expressing retinoic acid receptor beta 2 promotes recovery of function after corticospinal tract injury in the adult rat spinal cord. Hum Mol Genet 15:3107-3118. CrossRef Medline

Zhelyaznik N, Schrage K, McCaffery P, Mey J (2003) Activation of retinoic acid signalling after sciatic nerve injury: upregulation of cellular retinoid binding proteins. Eur J Neurosci 18:1033-1040. CrossRef Medline 